\title{
Relative critical exponents, non-vanishing and metrics with minimal singularities
}

\author{
Mihai Păun \\ Institut Élie Cartan, Nancy
}

\begin{abstract}
In this article we prove a non-vanishing statement, as well as several properties of metrics with minimal singularities of adjoint bundles. Our arguments involve many ideas from Y.-T. Siu's analytic proof of the finite generation of the canonical ring. An important technical tool is the notion of relative critical exponent of two closed positive currents with respect to a measure.
\end{abstract}

\section{$\S 0$ Introduction}

The main theme of this article is the notion of relative critical exponent of two closed positive currents with respect to a singular measure. Before presenting the results we obtain in connection with this definition, we recall the general context in [4], which will be relevant for us.

Let $X$ be a compact complex manifold and let $\Theta_{1}, \Theta_{2}$ be closed positive currents of $(1,1)$-type on $X$. We also consider a finite open covering $\left(U_{\alpha}\right)$ of $X$ and a family of functions $\psi:=\left(\psi_{\alpha}:=\psi_{\alpha}^{1}-\psi_{\alpha}^{2}\right)$ which are defined in terms of auxiliary functions $\psi_{\alpha}^{j}: U_{\alpha} \rightarrow[-\infty, \infty[$ and which have the properties :

- The difference $\psi_{\alpha}-\psi_{\beta}$ is non-singular on $U_{\alpha} \cap U_{\beta}$, and $\psi_{\alpha}^{j}$ is plurisubharmonic, for each $\alpha, \beta, j$;

-We have $\int_{U_{\alpha}} \exp \left(-\psi_{\alpha}\right) d \lambda<\infty$, for each $\alpha$, where $d \lambda$ the Lebesgue measure.

Then we define

$$
C_{\Theta_{1}, \psi}\left(X, \Theta_{2}\right):=\sup \left\{t \geq 0: \exp \left(t\left(\varphi_{\alpha}^{1}-\varphi_{\alpha}^{2}\right)-\psi_{\alpha}\right) \text { is in } L^{1}\left(U_{\alpha}\right), \forall \alpha\right\}
$$

where for each $j=1,2$, the function $\varphi_{\alpha}^{j}$ in the preceding expression is a local potential of $\Theta_{j}$.

The normalization we use in the definition of the potentials above is such that if $\Theta_{2}=[D], \Theta_{1}=0$ and $\psi=0$ (where $D$ is an effective $\mathbb{Q}$-divisor on $X$ ), we recover the usual notion of log-canonical threshold of $D$ in algebraic geometry. Thus, the quantity $C_{\Theta_{1}, \psi}\left(X, \Theta_{2}\right)$ is some kind of generalisation of that notion in algebraic geometry.

If $\left(\Theta_{j}, \psi\right)_{j=1,2}$ above have arbitrary singularities, it seems very difficult to say something meaningful about $C_{\Theta_{1}, \psi}\left(X, \Theta_{2}\right)$, i.e., this notion is far too general to work with. For example, it is not clear whether $C_{\Theta_{1}, \psi}\left(X, \Theta_{2}\right)$ is in general nonzero. Another problem would be to define a notion of multiplier sheaf in this context.

For these reasons, in the article [4] we are forced to confine ourselves most of the time to the logarithmic singularities case. An important idea in the proof of the next 
result is the consideration of a version of the threshold above which only takes into account a well chosen "logarithmic part" of the preceding currents/functions.

0.1 Theorem. Let $X$ be a projective manifold, and let $\theta_{L} \in \mathrm{NS}_{\mathbb{R}}(X)$ be a cohomology class in the real Neron-Severi space of $X$, such that:

(a) The adjoint class $c_{1}\left(K_{X}\right)+\theta_{L}$ is pseudoeffective, i.e. there exist a closed positive current

$$
\Theta_{K_{X}+L} \in c_{1}\left(K_{X}\right)+\theta_{L}
$$

(b) The class $\theta_{L}$ contains a Kähler current $\Theta_{L}$ such that for any index $\alpha$ we have

$$
e^{\left(1+\varepsilon_{0}\right)\left(\varphi_{K_{X}+L}-\varphi_{L}\right)} \in L^{1}\left(U_{\alpha}\right)
$$

where $\varepsilon_{0}$ is a positive real number, and $\varphi_{K_{X}+L}$ (resp. $\varphi_{L}$ ) is a local potential of the current $\Theta_{K_{X}+L}$ (resp. $\left.\Theta_{L}\right)$.

Then the adjoint class $c_{1}\left(K_{X}\right)+\theta_{L}$ contains an effective $\mathbb{R}$-divisor, i.e. there exist a finite family of positive reals $\mu^{j}$ and hypersurfaces $W_{j} \subset X$ such that

$$
\sum_{j=1}^{N} \mu^{j}\left[W_{j}\right] \in c_{1}\left(K_{X}\right)+\theta_{L}
$$

We use the subscript " $L$ " in the statement 0.1 in order to suggest that in some cases, $\theta_{L}$ is the Chern class of a line bundle $L$.

Firstly, we would like to mention that the above result generalizes the classical "non-vanishing" theorems of V. Shokurov and Y. Kawamata, cf. [23], [33], [34].

Secondly, it may seem that the integral hypothesis $(\star)$ in 0.1 is more general than the assumption that the critical exponent of $\Theta_{L}$ is greater than 1 (compare with [5]), but after suitable modification of $X$ one can see that it is enough to consider this case. This is a consequence of a theorem due to H. Skoda, see e.g. the paragraph 1.B.1 and 1.C ; however, we prefer to state our result in this form, since the hypothesis $(b)$ is almost canonical, in the sense that the quantity $(\star)$ with $\varepsilon_{0}=0$ is a global measure on $X$. We would also like to mention that the result in [5] is stronger : the authors obtain a statement within the numerical equivalence class rather than in cohomology. Of course, if $L$ is a $\mathbb{Q}$-bundle and $\theta_{L}$ is its Chern class, then 0.1 imply the existence of a section of some multiple of $K_{X}+L$; therefore, in the rational case we obtain the same non-vanishing theorem as in [5].

One important aspect of our proof is that is Char $p$-free, we avoid the explicit use of the minimal model program algorithm.

A theorem similar to 0.1 was proved by Y.-T. Siu in [39], pages 31-46. Even if the hypothesis in his statement are much more restrictive than in the theorem 0.1 , a substantial part of the arguments from his work will be used here.

It seems to us that 0.1 is not optimal : it is quite likely that one could prove a similar result with $\varepsilon_{0}=0$. Also, we expect our result to hold under weaker positivity assumptions on $\Theta_{L}$ : the best result one could hope would be to replace the assumption 
" $\Theta_{L}$ is a Kähler current" in $(b)$ above with the requirement $\Theta_{L} \geq 0$. However, the difficulties one has to deal with in this case appear to be rather severe.

Other possible generalization of 0.1 would be to work in purely transcendental setting i.e. without assuming that $\theta_{L} \in \mathrm{NS}_{\mathbb{R}}(X)$, but so far it is not clear what the statement should be.

Along the next lines we will give a very rough overview of our arguments ; as a starting point, we recall the classical non-vanishing result due to V. Shokurov (see [34]).

Theorem ([34]). Let $X$ be a projective manifold and let $D$, respectively $G=\sum_{j} \rho^{j} Z_{j}$ be a nef line bundle, resp. a $\mathbb{Q}$-divisor. We assume that the following relations hold:

(i) The $\mathbb{Q}$-divisor $D+G-K_{X}$ is nef and big;

(ii)The critical exponent of the current associated to $-G$ is greater than 1 .

Then for all large enough integers $m \in \mathbb{Z}_{+}$, the bundle $m D+\widehat{G}$ is effective, where $\widehat{G}$ is the "round-up" of $G$.

In order to explain the link between 0.1 and the above statement in a simple way, we assume for a moment the existence of a finite family of hypersurfaces $\left(Y_{j}\right)$ of $X$ with normal crossing intersections, such that

$$
\Theta_{L}=\sum_{j \in J} a_{L}^{j}\left[Y_{j}\right]+\Lambda_{L} \in \theta_{L}
$$

where $\Lambda_{L}$ is a Kähler metric, $J$ is a finite set and $a_{L}^{j}>0$. By the decomposition of closed positive currents theorem due to Y.-T. Siu (see [36]) we have

$$
\Theta_{K_{X}+L}=\sum_{j \in J} a_{K_{X}+L}^{j}\left[Y_{j}\right]+\sum_{i \in I} x^{i}\left[W_{i}\right]+\Lambda_{K_{X}+L} \in c_{1}\left(K_{X}\right)+\theta_{L} .
$$

The numbers $\left(x^{i}, a_{K_{X}+L}^{j}\right)$ in the formula above are positive reals and $\left(W_{i}\right)$ is a set of hypersurfaces of $X$ disjoint from $\left(Y_{j}\right)$. The cardinality of the index set $I$ could be infinite, and $\Lambda_{K_{X}+L}$ is a closed positive current whose Lelong level sets have codimension at least 2 .

Next, one can show that under the assumption $(\star)$ we have

$$
a_{K_{X}+L}^{j}-a_{L}^{j}>-1
$$

(see the section 1.C of this article). On the other hand, by the equality above we infer the following relation :

$$
\sum_{i \in I} x^{i}\left[W_{i}\right]+\Lambda_{K_{X}+L}+\sum_{j \in J}\left(a_{K_{X}+L}^{j}-a_{L}^{j}\right)\left[Y_{j}\right] \in c_{1}\left(K_{X}+\Lambda_{L}\right) .
$$

We introduce the following notations :

- $D^{\prime}:=\sum_{i} x^{i}\left[W_{i}\right]+\Lambda_{K_{X}+L}$

- $G^{\prime}:=\sum_{j \in J}\left(a_{K_{X}+L}^{j}-a_{L}^{j}\right)\left[Y_{j}\right]$ 
and the relation above shows that the Chern class of the $\mathbb{R}$-bundle $D^{\prime}+G^{\prime}-K_{X}$ contains a positive and non-singular representative, namely $\Lambda_{L}$. Moreover, since the coefficients of $G^{\prime}$ are greater than -1 , the hypothesis $(i i)$ of the above theorem is verified.

We assume further that the current $D^{\prime}$ above correspond to a nef line bundle, and that the coefficients $a_{K_{X}+L}^{j}, a_{L}^{j}$ are rational. Under these additional hypothesis, the theorem 0.1 is a direct consequence of the aforementioned result of V. Shokurov, as follows.

For each $j \in J$, we denote by $m^{j}$ the smallest integer greater than $a_{K_{X}+L}^{j}-a_{L}^{j}$ (i.e., the round-up of this difference). For any large enough integer $m \gg 0$, the theorem [34] show the existence of an effective $\mathbb{Q}$-section

$$
T_{m} \in c_{1}\left(D^{\prime}+\frac{1}{m} \widehat{G}^{\prime}\right)
$$

and we remark that

$$
T_{m}+\sum_{j \in J}\left(a_{K_{X}+L}^{j}-\frac{m^{j}}{m}\right) Y_{j} \in c_{1}\left(K_{X}\right)+\theta_{L}
$$

is effective, by the definition of $\left(m^{j}\right)$.

In this perspective, there are two important differences between 0.1 and the theorem of Shokurov. To start with, the currents $D^{\prime}$ and $G^{\prime}$ do not necessarily correspond to a line bundle, respectively to a $\mathbb{Q}$-line bundle. More seriously, the cohomology class of the current $D^{\prime}$ defined by the first bullet above may not be numerically effective. Actually, $D^{\prime}$ has two components : a "divisor-like" part -although the sum in question could be infinite- and a part (corresponding to $\Lambda_{K_{X}+L}$ ) which is nef in codimension 1 in the terminology [7]. The first component admits a well-defined restriction to any of the hypersurfaces $\left(Y_{j}\right)$, and the same thing is true for the second component, modulo a standard regularization process (see [7], [11]).

These excellent restriction properties of $D^{\prime}$ indicate that the proof of the theorem of Shokurov could eventually be adapted to the present setting. The principal use of the numerical effectivity of $D$ in [34] is to apply the vanishing theorems during an inductive process, so that twisted pluricanonical sections defined on some wellchosen hypersurface of $X$ extend to the whole manifold. In view of the very powerful extension theorems for the pluricanonical sections which were established since Y.T. Siu's invariance of plurigenera breakthrough (see [37], [38]), we show that indeed, despite a few serious technical difficulties, the general outline of the proof of the classical non-vanishing result still works.

Our article is organized as follows. We first review some standard facts concerning the notion of numerical dimension of a real $(1,1)$-class. If the dimension of $c_{1}\left(K_{X}\right)+\theta_{L}$ is equal to zero, then the theorem 0.1 is a consequence of a result due to S. Boucksom, see [7]. If this is not the case, we use the numerical positivity of $c_{1}\left(K_{X}\right)+\theta_{L}$, together with (a version of) the critical exponent defined above, in order to identify a hypersurface $S$ (the minimal center) of some modification of $X$ such that by restriction to $S$ we reproduce the same context as in 0.1 , except that the dimension drops (see 1.C and 
1.D). This part of our proof could be seen as a generalization of the classical arguments used in the Fujita conjecture literature (see [39] and the references therein).

During the restriction to the minimal center process, we will use in an essential manner the regularization techniques of Demailly ; a diophantine approximation argument is also involved, to reduce to the case where the geometric objects we are dealing with are rational (see 1.F, and also [5], [39]). Finally, we use the extension techniques of Siu and Hacon-McKernan adapted to the present situation (see 1.G and 1.H). The main technical point in $1 . \mathrm{H}$ is an ad hoc version of the invariance of plurigenera.

The important steps in our proof of the main theorem have their origin in the notes [39], [40] by Siu ; at the beginning of each concerned paragraph we will make this more explicit. Most of the subtle points in our arguments are equally observable in the algebraic geometry proof [5], as it was kindly explained to us by J. McKernan and S. Druel ; it would be very interesting to have a precise comparison between the two approaches.

The second part of this article is a corollary of the first. Suppose given a $\mathbb{Q}$-bundle $L$ which is big and endowed with a metric with positive curvature current whose critical exponent is greater than 1. Assume further that some multiple of the adjoint bundle $K_{X}+L$ is effective. In this context (in fact, in a slightly more general context, see section 2), we want to compare the metric with minimal singularities $\varphi_{\min }$ on the bundle $K_{X}+L$ with its algebraic approximations, i.e. induced by finite families of sections.

Very roughly, the main result we obtain is as follows. We assume that there exist an algebraic metric $\varphi_{\alpha}$ on $K_{X}+L$ which is strictly more singular than $\varphi_{\min }$; then we obtain a modification $\widehat{X} \rightarrow X$ and a new algebraic metric $\varphi_{\alpha^{\prime}}$ such that

$$
\nu_{S}\left(\varphi_{\alpha} \circ \mu\right)>\nu_{S}\left(\varphi_{\min } \circ \mu\right)
$$

and

$$
\nu_{S}\left(\varphi_{\alpha^{\prime}} \circ \mu\right)=\nu_{S}\left(\varphi_{\min } \circ \mu\right)
$$

for some hypersurface $S \subset \widehat{X}$. The new metric is produced using the non-vanishing theorem 0.1 .

Acknowledgments. It is a pleasure to thank S. Boucksom, B. Claudon and D. Varolin for their very interesting and pertinent comments about the present text, as well as for suggesting infinitely many improvements/short-cuts. Also, we would like to mention that this paper was completed during our visit to the Mittag-Leffler Institute; we are very grateful to the organizers for the invitation and support. We owe a debt of gratitude to J.-P. Demailly, S. Druel, L. Ein, J. McKernan and Y.-T. Siu, who shared with generosity and good humor their feelings about the topics in this paper. A substantial part of the techniques in this article emerged from our collaboration with B. Berndtsson; qu'il en soit chaleureusement remercié! 


\section{§1. A non-vanishing result}

In this section we are going to prove the theorem 0.1 , which is a version of the theorem obtained in [5] as a by-product of their fundamental result on the finiteness of the canonical ring (see equally [16], [25] for interesting presentations of [5]). To start with, we give some precisions about the notions which were involved in the statement 0.1.

As it is well-known, an integral cohomology class in $H^{2}(X, \mathbb{Z})$ is the Chern class of a holomorphic line bundle if and only if it is of $(1,1)$ type. The Neron-Severi group

$$
\mathrm{NS}(X):=H^{2}(X, \mathbb{Z}) \cap H^{1,1}(X, \mathbb{R})
$$

is the set of cohomology classes of line bundles. We denote by

$$
\mathrm{NS}_{\mathbb{R}}(X):=\mathrm{NS}(X) \otimes_{\mathbb{Z}} \mathbb{R} \subset H^{2}(X, \mathbb{R})
$$

the real Neron-Severi group.

We also recall the following notions.

Definition. A current $\Theta$ of type $(1,1)$ is called a Kähler current if there exist a Kähler metric $\omega$ on $X$ such that $\Theta \geq \omega$.

Definition. A function $\phi: X \rightarrow[-\infty, \infty[$ has logarithmic poles (or analytic singularities) if locally at each point $x \in X$ we have

$$
\phi=\log \left(\sum_{j}\left|f_{j}\right|^{2}\right)
$$

modulo $\mathrm{C}^{\infty}$ functions, where $f_{j} \in \mathcal{O}_{X, x}$ are local holomorphic functions.

Along the next lines we will use the proof of the classical case of the theorem 0.1 as a "guide", together with the theory of closed positive currents and the invariance of plurigenera. We borrow a few techniques from both analytic as well as algebraic works on the subject; however, we stress again that the characteristic $p$ methods, which seem to be essential in the later, are not used here.

\section{$\S 1$. A Numerical dimension of pseudoeffective line bundles}

Let $X$ be a compact Kähler manifold endowed with a metric $\omega$ and let $\alpha$ be a nonsingular $(1,1)$-form on $X$, which is assumed to be real and closed. We denote its cohomology class by $\{\alpha\} \in H^{1,1}(X, \mathbb{R})$, and we assume this class to be pseudoeffective.

Following [7], we denote by $\alpha[-\varepsilon \omega]$ the set of closed currents $T \in\{\alpha\}$ with logarithmic poles and such that

$$
T \geq-\varepsilon \omega
$$

The next fundamental result of J.-P. Demailly [11] is a quantitative version of the fact that if $\{\alpha\}$ is pseudoeffective, then for any $\varepsilon>0$ the set $\alpha[-\varepsilon \omega]$ is non empty. 
1.A.1 Theorem([11]). Let $T=\alpha+\sqrt{-1} \partial \bar{\partial} \varphi_{T}$ be a closed positive $(1,1)$-current on a compact complex manifold $X$ (here the function $\varphi_{T}$ is globally defined on $X$ ). Then for any real number $\varepsilon>0$ there exist a closed current

$$
T_{\varepsilon}:=\alpha+\sqrt{-1} \partial \bar{\partial} \varphi_{\varepsilon} \in \alpha[-\varepsilon \omega]
$$

such that we have the pointwise inequality $\varphi_{\varepsilon} \geq \varphi_{T}+\mathcal{O}(1)$.

In connection with his definition of mobile intersection of pseudoeffective classes, S. Boucksom proposed the next transcendental version of the classical notion of numerical dimension of a nef line bundle, as follows :

$$
\operatorname{nd}(\{\alpha\}):=\max \left\{k \in \mathbb{Z}_{+}: \lim \sup _{\varepsilon>0, T_{\varepsilon} \in \alpha[-\varepsilon \omega]} \int_{X \backslash Z_{\varepsilon}} T_{\varepsilon}^{k} \wedge \omega^{n-k}>0\right\}
$$

where $Z_{\varepsilon}$ above is the singular set of the current $T_{\varepsilon}$.

If $\{\alpha\}=c_{1}(L)$ for some nef line bundle $L \rightarrow X$, then $\operatorname{nd}(\{\alpha\})$ above become the usual numerical dimension of $L$; we refer to [7] for a more detailed account about this notion and its properties.

The statements which will follow assert the existence of geometric objects in the class $\{\alpha\}$ and its approximations, according to the size of its numerical dimension. The first one is due to S. Boucksom (see also the work of N. Nakayama, [31]).

1.A.2 Theorem ([7]). Let $\{\alpha\}$ be a $(1,1)$-cohomology class which is pseudoeffective and such that $\operatorname{nd}(\{\alpha\})=0$. Then there exist a closed positive current

$$
\Theta:=\sum_{j=1}^{\rho} \nu^{j}\left[Y_{j}\right] \in\{\alpha\}
$$

For a more complete discussion about the properties of the current $\Theta$ above we refer to the article [7].

Concerning the pseudoeffective classes $\{\alpha\} \in \mathrm{NS}_{\mathbb{R}}(X)$ whose numerical dimension is strictly greater than 0 , we have the following well-known statement.

1.A.3 Theorem. Let $X$ be a projective manifold, let $\{\alpha\} \in \mathrm{NS}_{\mathbb{R}}(X)$ be a pseudoeffective class, such that $\operatorname{nd}(\{\alpha\}) \geq 1$, and let $\beta$ be a Kähler current. Then for any $x \in X$ and $m \in \mathbb{Z}_{+}$there exist an integer $k_{m}$ and a closed positive current

$$
T_{k, x} \in\{m \alpha+\beta\}
$$

with logarithmic poles, and such that $\nu\left(T_{m, x}, x\right) \geq k_{m}$ and $k_{m} \rightarrow \infty$ as $m \rightarrow \infty$.

Proof. We fix an ample bundle $A \rightarrow X$, endowed with a metric $h$ with positive curvature. Let $N_{0} \in \mathbb{Z}_{+}$such that

$$
\beta \geq \frac{2}{N_{0}} \Theta_{h}(A)
$$


Since $\operatorname{nd}(\{\alpha\}) \geq 1$, there exist a positive constant $C>0$ and a family of currents $T_{\varepsilon} \in \alpha\left[-\varepsilon \Theta_{h}(A)\right]$ such that

$$
\int_{X \backslash Z_{\varepsilon}} T_{\varepsilon} \wedge \Theta_{h}(A)^{n-1} \geq C>0
$$

for any positive $\varepsilon$.

We will use now the hypothesis $\{\alpha\} \in \mathrm{NS}_{\mathbb{R}}(X)$ : there exist a sequence of bundles $\left(L_{m}\right)_{m \in \mathbb{Z}_{+}}$such that

$$
\left\|c_{1}\left(L_{m}\right)-m\{\alpha\}\right\| \rightarrow 0
$$

as $m \rightarrow \infty$, where $\|\cdot\|$ denotes any norm on $\mathrm{NS}_{\mathbb{R}}(X)$. We are not going to explain the details of this claim, since it is a simple diophantine approximation argument, see e.g. $[21],[5],[39]$, and the paragraph 1.F of this article, but rather indicate how to use the family of currents $T_{\varepsilon}$ above in order to obtain a lower bound of the quantity

$$
\frac{1}{p^{n}} h^{0}\left(X, p\left(N_{0} L_{m}+A\right)\right)
$$

as $p \rightarrow \infty$.

We recall that a very precise lower bound for the asymptotic behavior of the above dimension is provided by the holomorphic Morse inequalities, in the version obtained by L. Bonavero in [6]. In order to apply this result, we have to endow the bundle $N_{0} L_{m}+A$ with a suitable metric. To this end, we remark that we have

$$
N_{0} c_{1}\left(L_{m}\right)+c_{1}(A)=N_{0}\left(c_{1}\left(L_{m}\right)-m\{\alpha\}\right)+m N_{0}\{\alpha\}+c_{1}(A)
$$

The class $c_{1}\left(L_{m}\right)-m\{\alpha\}$ contains a non-singular representative $\rho_{m}$ which tend to zero as $m \rightarrow \infty$, by the relation (3). We take

$$
\varepsilon_{m}:=\frac{1}{2 m N_{0}}
$$

and then we have

$$
\rho_{m}+m N_{0} T_{\varepsilon_{m}}+\Theta_{h}(A) \geq 0
$$

if $m \gg 0$. The relation (4) imply the existence of a metric $h_{m}$ on the bundle $N_{0} L_{m}+A$, whose associated curvature current is (5).

By the holomorphic Morse inequalities [6] we obtain

$$
\frac{1}{p^{n}} h^{0}\left(X, p\left(N_{0} L_{m}+A\right)\right) \geq C_{0} \int_{X \backslash Z_{m}}\left(\rho_{m}+m N_{0} T_{\varepsilon_{m}}+\Theta_{h}(A)\right)^{n}
$$

where $Z_{m}$ is the set of singularities of $T_{\varepsilon_{m}}$ and $C_{0}$ is a positive constant, independent on $p$ and $m$. The inequality (2) show that the growth of the integral in the right hand side of the above inequality is at least linear with respect to $m$. 
Now we invoke the usual linear algebra arguments (see [28]) and infer the existence of a $\mathbb{Q}$-divisor $D_{m, x} \in c_{1}\left(N_{0} L_{m}+A\right)$ such that $\nu\left(D_{m, x}, x\right) \geq C m^{\frac{1}{n}}$ as $m \rightarrow \infty$. Then we define

$$
T_{m, x}:=\frac{1}{N_{0}}\left(\left[D_{m, x}\right]-\Theta_{h}(A)\right)+\beta-\rho_{m} ;
$$

it is a closed, positive $(1,1)$ current in the class $\{m \alpha+\beta\}$, and its Lelong number at $x$ tend to infinity with $m$. Thus the statement 1.A.3 is completely proved.

1.A.4 Remark. The theorem 1.A.3 holds in a more general context, as follows.

Theorem. Let $X$ be a compact Kähler manifold, endowed with a Kähler curent $\omega$, and let $\{\alpha\}$ be a pseudoeffective class, such that $\mathrm{nd}(\{\alpha\}) \geq 1$. Then for any $x \in X$ and $m \in \mathbb{Z}_{+}$there exist an integer $k_{m}$ and a closed positive current

$$
T_{m, x} \in\{m \alpha+\omega\}
$$

with logarithmic poles, and such that $\nu\left(T_{m, x}, x\right) \geq k_{m}$ and $k_{m} \rightarrow \infty$ as $m \rightarrow \infty$.

The proof of this result will not be discussed here, since we do not need it. Let us just mention that the "ancestor" of the above result can be found in the beautiful article [12] ; see also [15] for an overview of the techniques involved in the proof (the Yau theorem [46], and of the maximum principle of Bedford-Taylor [1]).

\section{$\S 1 . B$ Dichotomy}

After the preliminary discussion in the previous paragraph concerning the numerical dimension of the pseudoeffective classes and some of its properties, we start now the actual proof of the non-vanishing theorem. We denote by $\nu$ the numerical dimension of the class $c_{1}\left(K_{X}\right)+\theta_{L}$, and we proceed as in [5], [23], [27], [39].

- If $\nu=0$, then the existence of the $\mathbb{R}$-section in the class $c_{1}\left(K_{X}\right)+\theta_{L}$ is given by the theorem 1.A.2 above, therefore this first case is completely settled.

- The second case $\nu \geq 1$ is much more involved ; we are going to use induction on the dimension of the manifold. In order to ease the comprehension of our approach, we will first prove the theorem 0.1 under some additional rationality and finiteness assumptions in the next subsection.

\section{$\S 1 . B .1$ A particular case of 0.1}

The aim of the present subsection is to give a detailed proof of the next result, in order to illustrate the approach/difficulties for the general case.

1.B.1 Theorem. Let $X$ be a projective manifold and let $L \rightarrow X$ be a $\mathbb{Q}$-line bundle ; we denote by $\theta_{L} \in \mathrm{NS}_{\mathbb{R}}(X)$ its Chern class. We assume the existence of a closed positive current $\Theta_{K_{X}+L} \in c_{1}\left(K_{X}\right)+\theta_{L}$ with logarithmic poles and rational Lelong numbers. Moreover, we assume that the class $\theta_{L}$ contains a Kähler current $\Theta_{L}$ such that

$$
\int_{X} \exp \left(\varphi_{K_{X}+L}-\varphi_{L}\right) d \lambda<\infty .
$$


Then $H^{0}\left(X, p\left(K_{X}+L\right)\right) \neq 0$ for all $p$ large and divisible enough.

We remark that the integral condition above is less restrictive than the hypothesis in the statement 0.1 ; therefore, the (heavy) additional assumption is the existence of a current with log poles and rational singularities in the class $\left\{K_{X}+L\right\}$.

Proof. In the first place, we would like to mention that this version of the non-vanishing is almost due to Shokurov, but we are going to prove it in a slightly different manner, which is better adapted for the illustration of the general case.

To start with, we remark that we can assume that the current $T_{m}$ in the statement 1.A.3 is given by an effective $\mathbb{Q}$-section $D$ of the bundle $m\left(K_{X}+L\right)+L$. Indeed, as a consequence of the holomorphic Morse inequalities we have

$$
\frac{1}{p^{n}} h^{0}\left(X, m p\left(K_{X}+L\right)+p L\right) \geq C m^{\nu}
$$

see e.g. [6]. Thus, the existence of the section $D$ above is provided by the usual linear algebra arguments. We fix $m$ large enough, so the the singularity of $\nu\left(D, x_{0}\right) \geq n+1$, where the point $x_{0}$ is chosen such that $\Theta_{K_{X}+L}$ and $\Theta_{L}$ are non-singular at $x_{0}$.

Now let us consider the following relative critical exponent, which is adapted to the current situation. We set

$$
\tau:=C_{m \Theta_{K_{X}+L}+\Theta_{L}, e^{\varphi_{K}+L}-\varphi_{L}}(X, D) ;
$$

in other words we have

$$
\tau=\sup \left\{t \in \mathbb{R}_{+}: \int_{X} e^{t\left(\varphi_{L}+m \varphi_{K_{X}+L}-\varphi_{D}\right)} e^{\varphi_{K_{X}+L}-\varphi_{L}} d \lambda<\infty\right\} .
$$

Thus we consider the relative critical exponent with respect to the singular measure of finite mass

$$
e^{\varphi_{K}+L-\varphi_{L}} d \lambda
$$

We note that we have the relations

$$
0<\tau<1
$$

indeed, the first inequality is due to the fact that by hypothesis the relative critical exponent of $\Theta_{L}$ with respect to $\Theta_{K_{X}+L}$ is greater than 1. As for the second one, it can be seen as a consequence of the fact that the singularity of $D$ et $x_{0}$ is large enough.

As in the proof of the non-vanishing result in [34], we are going to use an appropriate modification of the manifold $X$ in order to have an interpretation of the quantity $\tau$.

Let $\mu: \widehat{X} \rightarrow X$ be a modification with such that the singular part of the inverse images of the currents below have normal crossing.

$$
\mu^{\star}\left(\Theta_{K_{X}+L}\right)=\sum_{j \in J} a_{K_{X}+L}^{j}\left[Y_{j}\right]+\widehat{\Lambda}_{K_{X}+L}
$$




$$
\begin{gathered}
\mu^{\star}\left(\Theta_{L}\right)=\sum_{j \in J} a_{L}^{j}\left[Y_{j}\right]+\widehat{\Lambda}_{L} \\
\mu^{\star}\left(\Theta_{D}\right)=\sum_{j \in J} a_{D}^{j}\left[Y_{j}\right] \\
K_{\widehat{X} / X}=\sum_{j \in J} a_{\widehat{X} / X}^{j}\left[Y_{j}\right]
\end{gathered}
$$

where $J$ is a finite set, $\left(a^{j}\right)$ are non-negative real numbers (some of them may be zero, since we use the same family of indexes), $\left(Y_{j}\right)$ are divisors in $\widehat{X}$, either proper transforms of divisors in $X$ or $\mu$-exceptional ; we will assume that they contain all the exceptional divisors of $\mu$ (as we can take the corresponding coefficients zero if necessary). Finally $\widehat{\Lambda}$ are non-singular, semi-positive $(1,1)$-forms on $\widehat{X}$. Moreover, we remark that

$$
\widehat{\Lambda}_{L} \geq \mu^{\star} \omega \text {. }
$$

Let $\omega$ (respectively $\widehat{\omega}$ ) be a Kähler metric on $X$ (respectively $\widehat{X}$ ). The definition of our relative critical exponent (6) show that the quantity we have to evaluate is

$$
\mu^{\star}\left(\Theta_{\omega}\left(K_{X}\right)+t\left([D]-m \Theta_{K_{X}+L}-\Theta_{L}\right)+\Theta_{L}-\Theta_{K_{X}+L}\right)
$$

that is to say

$$
\mu^{\star}\left(\Theta_{\omega}\left(K_{X}\right)+t[D]-(1+t m) \Theta_{K_{X}+L}+(1-t) \Theta_{L}\right)
$$

where we denote by $[D]$ the current of integration associated to the $\mathbb{Q}$-divisor $D$.

We first remark that the cohomology class of the current above is equal to zero. This is an important point in our proof.

On the other hand, the equalities (8)-(11) show that for any positive real $t$ we have

$$
\begin{aligned}
& \mu^{\star}\left(\Theta_{\omega}\left(K_{X}\right)+t[D]+(1-t) \Theta_{L}-(1+m t) \Theta_{K_{X}+L}\right) \equiv \\
& \quad \equiv \Theta_{\widehat{\omega}}\left(K_{\widehat{X}}\right)+\sum_{j \in J}\left(t a_{D}^{j}+(1-t) a_{L}^{j}-(1+m t) a_{K_{X}+L}^{j}-a_{\widehat{X} / X}^{j}\right)\left[Y_{j}\right]+ \\
& \quad+(1-t) \widehat{\Lambda}_{L}-(1+m t) \widehat{\Lambda}_{K_{X}+L}
\end{aligned}
$$

where the symbol $\equiv$ means that the two currents above have the same cohomology class. Therefore, we get the next cohomological identity

$$
(1+m t) \widehat{\Lambda}_{K_{X}+L} \equiv \Theta_{\widehat{\omega}}\left(K_{\widehat{X}}\right)+\sum_{j \in J}\left(t a_{D}^{j}+(1-t) a_{L}^{j}-(1+m t) a_{K_{X}+L}^{j}-a_{\widehat{X} / X}^{j}\right)\left[Y_{j}\right]+(1-t) \widehat{\Lambda}_{L}
$$


By the definition (6), for any real $t<\tau$ and $j \in J$ we have the following inequalities : $(\bullet)_{j} t\left(a_{D}^{j}-a_{L}^{j}-m a_{K_{X}+L}^{j}\right)<1+a_{K_{X}+L}^{j}-a_{L}^{j}+a_{\widehat{X} / X}^{j}$.

In addition at least one of the previous inequality is in fact an equality for $t=\tau$. We will show next that we can further modify the inverse image of the current $\Theta_{L}$ in order to have equality for precisely on single index ; we stress on the fact that we can achieve this without changing the cohomology classes above, since $L$ is big.

To this end, we remark that given any family of positive rational numbers $\eta^{j}$, the $\mathbb{Q}$-divisor

$$
\sum_{j \in J}\left(a_{L}^{j}+\eta^{j}\right)\left[Y_{j}\right]+\widehat{\Lambda}_{L, 1}
$$

belongs to the Chern class of the $\mathbb{Q}$-line bundle $\mu^{\star}(L)$, provided that

$$
\widehat{\Lambda}_{L, 1} \equiv \widehat{\Lambda}_{L}-\sum_{j \in J} \eta^{j}\left[Y_{j}\right]
$$

Now we recall that for any Kähler metric $\omega$ on $X$, the cohomology class of the next current

$$
\mu^{\star}(\omega)-\sum_{j \in J} \eta^{j}\left[Y_{j}\right]
$$

contains a positive representative, for some family of positive rational numbers $\left(\eta^{j}\right)$, which can be chosen as small as we want. Here we use the fact that all the $\mu$-exceptional divisors are among the $\left(Y_{j}\right)$ above ; as for the non-exceptional ones, they are absorbed by $\omega$. We remark that once such a family is fixed, any small enough perturbation of it will have the same properties. Therefore we can assume that all the rational numbers

$$
\frac{1+a_{K_{X}+L}^{j}+a_{\widehat{X} / X}^{j}-a_{L}^{j}}{a_{D}^{j}-a_{L}^{j}-m a_{K_{X}+L}^{j}}
$$

are distinct, where the $a_{L}^{j}$ in the above quotient is the coefficient of $\nu^{\star} \Theta_{L}$ after perturbation. In what will follow, we still use the same notations for the inverse image of $L$, but we keep in mind that $\widehat{\Lambda}_{L}$ is now a Kähler metric on $\widehat{X}$.

Our $L^{2}$ condition in the theorem 1.B.1 is translated via the blow up as follows

$$
1+a_{K_{X}+L}^{j}+a_{\widehat{X} / X}^{j}-a_{L}^{j}>0
$$

for any index $j \in J$, therefore we have the inequalities $(\bullet)_{j}$ above are automatically satisfied for the indexes $j$ such that

$$
a_{D}^{j}-a_{L}^{j}-m a_{K_{X}+L}^{j} \leq 0 .
$$

Therefore we obtain

$$
\tau=\frac{1+a_{K_{X}+L}^{j}+a_{\widehat{X} / X}^{j}-a_{L}^{j}}{a_{D}^{j}-a_{L}^{j}-m a_{K_{X}+L}^{j}}
$$


for a unique $j=j_{0}$; we remark that $\tau \in \mathbb{Q}$, by our rationality hypothesis. We will denote by $S:=Y_{j_{0}}$.

In conclusion, we have the numerical identity

$$
K_{\widehat{X}}+S+\widehat{L} \equiv \widehat{\Theta}
$$

where $\widehat{L}$ is any $\mathbb{Q}$-bundle on $\widehat{X}$ whose associated Chern class contains the current

$$
\sum_{j \in J_{p}}\left(\tau a_{D}^{j}+(1-\tau) a_{L}^{j}-(1+m \tau) a_{K_{X}+L}^{j}-a_{\widehat{X} / X}^{j}\right)\left[Y_{j}\right]+(1-\tau) \widehat{\Lambda}_{L}
$$

We denote by

$$
\widehat{\Theta}:=\sum_{j \in J_{n}}\left((1+m \tau) a_{K_{X}+L}^{j}+a_{\widehat{X} / X}^{j}-\tau a_{D}^{j}-(1-\tau) a_{L}^{j}\right)\left[Y_{j}\right]+(1+m \tau) \Lambda_{K_{X}+L} .
$$

The $J_{p}$ (respectively $J_{n}$ ) are the sets of indexes $j \in J$ for which the corresponding coefficient of $Y_{j}$ in the expression (16) and in the definition of $\widehat{\Theta}$ is positive ; we remark that we have

$$
J=J_{p} \cup J_{n} \cup\left\{j_{0}\right\}
$$

It is at this point that one can see the utility of the relative critical exponent defined above : the $\mathbb{Q}$-bundle $\widehat{L}$ and its restriction to $S$ are big, and that the coefficients of their respective singular part are strictly smaller than 1 . Also, the numerical identity (15) restricted to $S$ show that $K_{S}+\widehat{L}_{\mid S}$ is pseudoeffective, and it has a metric with analytic singularities, since we have

$K_{S}+\widehat{L}_{\mid S} \equiv \sum_{j \in J_{n}}\left((1+m \tau) a_{K_{X}+L}^{j}+a_{\widehat{X} / X}^{j}-\tau a_{D}^{j}-(1-\tau) a_{L}^{j}\right)\left[Y_{j \mid S}\right]+(1+m \tau) \Lambda_{K_{X}+L \mid S}$

Therefore by induction we infer that

$$
H^{0}\left(S, p\left(K_{S}+\widehat{L}_{\mid S}\right)\right) \neq 0
$$

for all large and divisible $p$.

We claim next that any section

$$
u \in H^{0}\left(S, p\left(K_{S}+\widehat{L}_{\mid S}\right)\right)
$$

admits an extension $U$ to $\widehat{X}$. This is a immediate application of the invariance of plurigenera of Siu, in the version due to Hacon-McKernan [19]. Indeed, we have :

1. The bundle $\widehat{L}$ is decomposed as sum of an ample line bundle and an effective one with critical exponent greater than 1 ;

2. The bundle $K_{\widehat{X}}+S+\widehat{L}$ admit a metric with positive curvature, whose singular part is transversal to the support of the effective part of $\widehat{L}$. 
Thus by the extension theorem in [19] we obtain $U \in H^{0}\left(\widehat{X}, p\left(K_{\widehat{X}}+S+\widehat{L}\right)\right)$ such that $U_{\mid S}=u$.

A last observation is that since $0<\tau<1$ we have

$$
\mu^{\star}(\tau D+(1-\tau) L)=S+\widehat{L}+F
$$

where some multiple of $F$ has non-zero sections. Indeed, by the formulas (16) and (8)-(11) we have

$$
F=\sum_{j \in J_{p} \cup\left\{j_{0}\right\}}\left((1+m \tau) a_{K_{X}+L}^{j}+a_{\widehat{X} / X}^{j}\right)\left[Y_{j}\right]+\sum_{j \in J_{n}}\left(\tau a_{D}^{j}+(1-\tau) a_{L}^{j}\right)\left[Y_{j}\right]
$$

Now we twist an appropriate power of $U$ with the section of the corresponding multiple of $F$ and we obtain a section in a multiple of the bundle

$$
K_{X}+\tau\left(L+m\left(K_{X}+L\right)\right)+(1-\tau) L
$$

which is nothing but $(1+\tau m)\left(K_{X}+L\right)$.

Therefore, the particular case of the theorem 0.1 is completely proved.

Remark. The only difference between the theorem 1.B.1 and the result of Shokurov in [34] is the existence of the singular part $\left(Y_{j}\right)$ in the expression of $\widehat{L}$ and $\widehat{\Theta}$ above. However, the singular part encoded by $\widehat{L}$ is small (the coefficients are smaller than 1) and the one inside $\widehat{\Theta}$ is transversal to the singularities of $\widehat{L}$ : it is precisely for this reason that the invariance of plurigenera [17] still holds, and it is used to replace the Kawamata-Viehweg vanishing theorem (cf. [22], [45], [30]) to give the desired result.

\section{§1.C Relative threshold of the logarithmic part of $\Theta_{K_{X}+L}$}

We continue our proof of the general case of 0.1 by introducing a version of the relative threshold which is adapted to the case where $\Theta_{K_{X}+L}$ does not necessarily have log poles. The main motivation is that we still want to use the same approach as in the previous section and settle the general case by induction.

We recall that we have $\operatorname{nd}\left(\left\{K_{X}+L\right\}\right) \geq 1$. Let $x_{0} \in X$ be a very general point, such that $\nu\left(\Theta_{K_{X}+L}, x_{0}\right)=0$. By the theorem 1.A.3, there exist a closed positive current

$$
T \in m\left(c_{1}\left(K_{X}\right)+\theta_{L}\right)+\theta_{L}
$$

with logarithmic poles, such that $\nu\left(T, x_{0}\right)>n+1$ (the positive integer $m$ above is large and fixed during the rest of the proof). We remark that $T$ is the substitute for the $\mathbb{Q}$-section $D$ in the previous paragraph. 
Let $\mu_{0}: \widetilde{X} \rightarrow X$ be a common log resolution of the currents $T$ and $\Theta_{L}$. By this we mean that $\mu_{0}$ is the composition of a sequence of blow-up maps with non-singular centers, such that we have

$$
\begin{gathered}
\mu_{0}^{\star}\left(\Theta_{L}\right)=\sum_{j \in J} a_{L}^{j}\left[Y_{j}\right]+\widetilde{\Lambda}_{L} \\
\mu_{0}^{\star}(T)=\sum_{j \in J} a_{T}^{j}\left[Y_{j}\right]+\widetilde{\Lambda}_{T} \\
K_{\widetilde{X} / X}=\sum_{j \in J} a_{\widetilde{X} / X}^{j}\left[Y_{j}\right]
\end{gathered}
$$

where the divisors above are assumed to be non-singular and to have normal crossings.

We remark that the existence of the manifold $\widetilde{X}$ respectively of the map $\mu_{0}$ is a consequence of the fact that the currents $T$ and $\Theta_{L}$ have $\log$ poles.

Now the current $\Theta_{K_{X}+L}$ enter into the picture. Let us consider its inverse image via the map $\mu_{0}$ :

$$
\mu_{0}^{\star}\left(\Theta_{K_{X}+L}\right)=\sum_{j \in J} a_{K_{X}+L}^{j}\left[Y_{j}\right]+\widetilde{\Lambda}_{K_{X}+L}
$$

where $\widetilde{\Lambda}_{K_{X}+L}$ in the relation above is a closed positive current, such that

$$
\nu_{Y_{j}}\left(\widetilde{\Lambda}_{K_{X}+L}\right)=0
$$

for all $j \in J$ (the decomposition (22) is a direct consequence of a result due to Siu in $[36])$.

In other words, even if $\widetilde{\Lambda}_{K_{X}+L}$ is not smooth anymore (as it was the case in the previous paragraph), its generic Lelong number along all the possible candidates for the hypersurface $S$ in the previous section is zero.

Let us denote by $\widetilde{D}$ the first (divisor-type) part of the current $\mu^{\star}\left(\Theta_{K_{X}+L}\right)$; we consider the next quantity

$$
\tau:=\sup \left\{t \in \mathbb{R}_{+}: \int_{\widetilde{X}} \exp \left(t\left(\varphi_{L} \circ \mu_{0}+m \varphi_{\widetilde{D}}-\varphi_{T} \circ \mu_{0}\right)+\varphi_{\widetilde{D}}+\varphi_{\widetilde{X} / X}-\varphi_{L} \circ \mu_{0}\right) d \lambda<\infty\right\}
$$

We will prove next that we have the relations

$$
0<\tau<1
$$


The latter inequality is due to the fact that the singularity of $T$ at $x_{0}$ is large, and moreover $\mu_{0}^{-1}\left(x_{0}\right)$ is disjoint from the support of $\widetilde{D}$ (thus, it is here that the choice of a very generic point $x_{0}$ is important). As for the former inequality, we remark that by hypothesis we have

$$
\int_{\widetilde{X}} \exp \left(\left(1+\varepsilon_{0}\right)\left(\varphi_{K_{X}+L} \circ \mu_{0}-\varphi_{L} \circ \mu_{0}\right)+\varphi_{\widetilde{X} / X}\right) d \lambda<\infty
$$

so in particular

$$
I:=\int_{\widetilde{X}} \exp \left(\left(1+\varepsilon_{0}\right)\left(\varphi_{\widetilde{D}}+\varphi_{\widetilde{\Lambda}}-\varphi_{L} \circ \mu_{0}+\varphi_{\widetilde{X} / X}\right)\right) d \lambda<\infty
$$

We consider a point $y \in \widetilde{X}$ such that

$$
\nu\left(\widetilde{\Lambda}_{K_{X}+L}, y\right)=0
$$

and we have

$$
\int_{(\widetilde{X}, y)} \exp \left(\varphi_{\widetilde{D}}-\varphi_{L} \circ \mu_{0}+\varphi_{\widetilde{X} / X}\right) d \lambda \leq I^{1 / 1+\varepsilon_{0}}\left(\int_{(\widetilde{X}, y)} \exp \left(-\left(1+1 / \varepsilon_{0}\right) \varphi_{\widetilde{\Lambda}}\right) d \lambda\right)^{\varepsilon_{0} / 1+\varepsilon_{0}}
$$

and the last integral is convergent since the Lelong number of $\widetilde{\Lambda}_{L}$ at $y$ is equal to zero, therefore we can apply the theorem of Skoda, see [35].

In conclusion, the function $\exp \left(\varphi_{\widetilde{D}}-\varphi_{L} \circ \mu_{0}+\varphi_{\widetilde{X} / X}\right)$ is in $L^{1}$ at the generic point of each $Y_{j}$, by the relation (18). Together with the fact that the hypersurfaces $\left(Y_{j}\right)$ have normal crossing, this prove that $\tau>0$.

As before, we can obtain the explicit expression of the threshold $\tau$ (modulo perturbation) by using the modification $\mu_{0}$ as follows. Given any real number $t$, we have

$$
\begin{aligned}
\mu^{\star}\left(\Theta_{\omega}\left(K_{X}\right)+t\left(T-\Theta_{L}\right)+\Theta_{L}\right) \equiv \Theta_{\widehat{\omega}}\left(K_{\widetilde{X}}\right)+(1+m t) \widetilde{D}+(1-t) \widetilde{\Lambda}_{L}+t \widetilde{\Lambda}_{T} \\
+\sum_{j \in J}\left(t a_{T}^{j}+(1-t) a_{L}^{j}-(1+m t) a_{K_{X}+L}^{j}-a_{\widetilde{X} / X}^{j}\right)\left[Y_{j}\right] .
\end{aligned}
$$

We have $\mu^{\star}\left(\Theta_{K_{X}+L}\right)=\widetilde{D}+\widetilde{\Lambda}_{K_{X}+L}$, and on the other hand the cohomology class of the current

$$
t\left(T-\Theta_{L}\right)+\Theta_{L}-(1+m t) \Theta_{K_{X}+L}
$$

is equal to the first Chern class of $X$, so the previous relation can be written as

$$
\begin{aligned}
(1+m t) \widetilde{\Lambda}_{K_{X}+L} \equiv & \Theta_{\widehat{\omega}}\left(K_{\widetilde{X}}\right)+\sum_{j \in J}\left(t a_{T}^{j}+(1-t) a_{L}^{j}-(1+m t) a_{K_{X}+L}^{j}-a_{\widetilde{X} / X}^{j}\right)\left[Y_{j}\right]+ \\
& (1-t) \widetilde{\Lambda}_{L}+t \widetilde{\Lambda}_{T} .
\end{aligned}
$$


The perturbation argument used in the previous paragraph is still valid ; in conclusion, for $t:=\tau$ (or better say, a slight modification of this quantity, since we change the inverse image of the current $\Theta_{L}$ within the same cohomology class) we can assume that we have

$$
(1+m \tau) \widetilde{\Lambda}_{K_{X}+L}+\widetilde{G} \equiv c_{1}\left(K_{\widetilde{X}}+\widetilde{S}\right)+\theta_{\widetilde{L}}
$$

where the notations we use are as follows :

$$
\begin{aligned}
\theta_{\widetilde{L}} & : \equiv \sum_{j \in J_{p}}\left(\tau a_{T}^{j}+(1-\tau) a_{L}^{j}-(1+m \tau) a_{K_{X}+L}^{j}-a_{\widetilde{X} / X}^{j}\right)\left[Y_{j}\right]+ \\
& +(1-\tau) \widetilde{\Lambda}_{L}+\tau \widetilde{\Lambda}_{T}
\end{aligned}
$$

as well as

$$
\widetilde{G}:=\sum_{j \in J_{n}}\left((1+m \tau) a_{K_{X}+L}^{j}+a_{\widetilde{X} / X}^{j}-\tau a_{T}^{j}-(1-\tau) a_{L}^{j}\right)\left[Y_{j}\right]
$$

after perturbation, we can assume that $\widetilde{\Lambda}_{L}$ is a Kähler metric. In the above relations, we have used the same conventions as in 1.B for the definition of $J_{p}, J_{n}$.

\section{§1.D Properties of $\boldsymbol{\theta}_{\widetilde{\boldsymbol{L}}}$}

We collect in this paragraph the main features of the class $\theta_{\widetilde{L}}$ which will be needed later.

- In the first place, by the definition of the threshold $\tau$, we see that the coefficients of the singular part in the expression $(26)$ are strictly smaller than 1 . Thus, $\theta_{\widetilde{L}}$ contains a closed positive current whose critical exponent is strictly greater than 1 ; it is equally a Kähler current, as it dominates $(1-\tau) \widetilde{\Lambda}_{L}$, and this form is positive definite on $\tilde{X}$.

- There exist an effective $\mathbb{R}$-divisor $\Delta$ such that

$$
\begin{aligned}
\theta_{\widetilde{L}}+\{\widetilde{S}+\Delta\} & =\mu_{0}^{\star}\left(\tau\{T\}+(1-\tau) \theta_{L}\right) \equiv \\
& \equiv \mu_{0}^{\star}\left(\theta_{L}+\tau m\left(c_{1}\left(K_{X}\right)+\theta_{L}\right)\right)
\end{aligned}
$$

(the precise expression of $\Delta$ is not relevant for the moment, but one can easily get it from the relations (25), (26) and (27) above).

Therefore, it is enough to produce an effective $\mathbb{R}$-divisor in the cohomology class $c_{1}\left(K_{\widetilde{X}}+\widetilde{S}\right)+\theta_{\widetilde{L}}$ in order to complete the proof of the theorem 0.1.

- The adjoint class $c_{1}\left(K_{\widetilde{X}}+\widetilde{S}\right)+\theta_{\widetilde{L}}$ is pseudoeffective ; moreover, it contains the closed positive current

$$
(1+m \tau) \widetilde{\Lambda}_{K_{X}+L}+\widetilde{G}
$$

whose Lelong number at the generic point of $\widetilde{S}$ is equal to zero. 
- By using a sequence of blow-up maps, we can even assume that the components $\left(Y_{j}\right)_{j \in J_{p}}$ of the representative $(26)$ of $\theta_{\widetilde{L}}$ have empty mutual intersections. Indeed, this is a simple -but nevertheless crucial!- result due to Hacon-McKernan, which we recall next.

We denote by $B$ an effective $\mathbb{Q}$-divisor, whose support do not contain $\widetilde{S}$, such that Supp $B \cup \widetilde{S}$ has normal crossings and such that its coefficients are strictly smaller than 1.

Lemma ([20]). There exist a birational map $\mu_{1}: \widehat{X} \rightarrow \widetilde{X}$ such that

$$
\mu_{1}^{\star}\left(K_{\widetilde{X}}+\widetilde{S}+B\right)+E_{\widehat{X}}=K_{\widehat{X}}+S+\Gamma
$$

where $E_{\widehat{X}}$ and $\Gamma$ are effective with no common components, $E_{\widehat{X}}$ is exceptional and $S$ is the proper transform of $\widetilde{S}$; moreover, the support of the divisor $\Gamma$ has normal crossings, its coefficients are strictly smaller than 1 and the intersection of any two components is empty.

The proof of the above lemma is by induction on the number of the components of $B$ having non-empty intersection ; the sets which we blow up to obtain $\mu_{1}$ are precisely the said intersections. Since from the start the components of $B$ have normal crossings, the restriction of the map $\mu_{1}$ to the proper transform $S$ of $\widetilde{S}$ will be isomorphic at the generic point of $S$.

In our setting the $\mathbb{R}$-divisor $B$ above is defined as

$$
B:=\sum_{j \in J_{p}}\left(\tau a_{T}^{j}+(1-\tau) a_{L}^{j}-(1+m \tau) a_{K_{X}+L}^{j}-a_{\widetilde{X} / X}^{j}\right) Y_{j}
$$

and we have

$$
\mu_{1}^{\star}\left(c_{1}\left(K_{\widetilde{X}}+\widetilde{S}\right)+\left\{[B]+(1-t) \widetilde{\Lambda}_{L}+t \widetilde{\Lambda}_{T}\right\}\right)+\left\{E_{\widehat{X}}\right\}=c_{1}\left(K_{\widehat{X}}+S\right)+\theta
$$

where the cohomology class $\theta$ above contain a representative which can be written as follows

$$
\sum_{j \in I} \rho^{j} W_{j}+\widehat{\Lambda}
$$

where $0<\rho^{j}<1$ for any $j$, the hypersurfaces $W_{j}$ are non-singular and $W_{j} \cap W_{k}=\emptyset$ if $j \neq k$, and $\widehat{\Lambda}$ is smooth, semipositive, whose restriction to $S$ is positively defined at the generic point.

In addition, we remark that the new modification $\mu_{1}$ does not affect the bullets above. We summarize the discussion in this paragraph in the next statement (in which we equally adjust the notations).

1.D.1 Proposition. There exist a birational map $\mu: \widehat{X} \rightarrow X$ and a class $\theta_{\widehat{L}} \in \mathrm{NS}_{\mathbb{R}}(\widehat{X})$ which contain the current

$$
\sum_{j \in J} \nu^{j} Y_{j}+\widehat{\Lambda}_{L}
$$


where $0<\nu^{j}<1$, the hypersurfaces $Y_{j}$ above are smooth, they have empty mutual intersection and moreover the following hold:

i) There exist a closed positive $(1,1)$-current $\Theta$ on $\widehat{X}$ with the property that

$$
\Theta \in c_{1}\left(K_{\widehat{X}}+S\right)+\theta_{\widehat{L}}
$$

where $S \subset \widehat{X}$ is a non-singular hypersurface which has transversal intersections with $\left(Y_{j}\right)$;

ii) The support of the divisorial part of the current $\Theta$ is disjoint from the set $\left(S, Y_{j}\right)$;

iii) There exist a map $\mu_{1}: \widehat{X} \rightarrow \widetilde{X}$ such that $S$ is not $\mu_{1}$-exceptional, and such that $\widehat{\Lambda}_{L}$ is greater than the inverse image of a Kähler metric on $\widetilde{X}$ via $\mu_{1}$. Therefore, the form $\widehat{\Lambda}_{L}$ is positive defined at the generic point of $\widehat{X}$, and so is its restriction to the generic point of $S$;

iv) There exist an effective $\mathbb{R}$-divisor $\Delta$ on $\widehat{X}$ such that

$$
\theta_{\widehat{L}}+\{S+\Delta\}=\mu^{\star}\left(\theta_{L}+\tau m\left(c_{1}\left(K_{X}\right)+\theta_{L}\right)\right)+\{E\}
$$

where $E$ is $\mu$-exceptional.

We remark that the relation $i$ i) imply that the Lelong number of $\Theta$ at the generic point of $S$ is equal to zero but nevertheless, the local potentials of $\Theta$ may be identically $-\infty$ when restricted to $S$. Therefore in order to be able to use the induction hypothesis, we have to regularize it. Certainly this creates some difficulties, which we overcome along the next paragraphs.

\section{§1.E Regularization and induction}

We consider now the family of approximations $\left(\Theta_{\varepsilon}\right)_{\varepsilon>0}$ of the current $\Theta$ given by the theorem 1.A.1. For each $\varepsilon>0$, the current $\Theta_{\varepsilon}$ has $\log$ poles, and we equally have

$$
\nu\left(\Theta_{\varepsilon}, x\right) \leq \nu(\Theta, x)
$$

for any $x \in \widetilde{X}$. In particular, we have $\nu\left(\Theta_{\varepsilon}, x\right)=0$ for the generic point $x \in S$; in other words, the restriction of the current $\Theta_{\varepsilon}$ to $S$ is well defined (i.e. its potential is not identically equal to $-\infty$ at each point of $S$ ). Therefore, since the regularization process does not change the cohomology class, the relation $i$ ) of the proposition 1.D.1 above imply

$$
\Theta_{\varepsilon \mid S} \in c_{1}\left(K_{S}\right)+\theta_{\widehat{L} \mid S}
$$

Next, we have the following decomposition

$$
\Theta_{\varepsilon \mid S}=\sum_{j \in J} \rho^{\varepsilon, j} Y_{j \mid S}+R_{\varepsilon}
$$


where the coefficients $\left(\rho^{\varepsilon, j}\right)$ are positive real numbers, the generic Lelong number of $R_{\varepsilon}$ along $Y_{j} \cap S$ is zero, and moreover we have

$$
R_{\varepsilon} \geq-\varepsilon \omega_{\mid S}
$$

We remark that this current may be singular along some other hypersurfaces of $S$.

For each index $j \in J$ we will assume that the next limit

$$
\rho^{\infty, j}:=\lim _{\varepsilon \rightarrow 0} \rho^{\varepsilon, j}
$$

exist, and we introduce the following notation

$$
I:=\left\{j \in J: \rho^{\infty, j} \geq \nu^{j}\right\}
$$

The numerical identity (28) coupled with (29) show that we have

$$
\sum_{j \in I}\left(\rho^{\infty, j}-\nu^{j}\right)\left[Y_{j \mid S}\right]+R_{\varepsilon}+\sum_{j \in J}\left(\rho^{\varepsilon, j}-\rho^{\infty, j}\right)\left[Y_{j \mid S}\right] \in c_{1}\left(K_{S}\right)+\theta_{L_{S}}
$$

where $\theta_{L_{S}}$ is the cohomology class of the current

$$
\sum_{j \in J \backslash I}\left(\nu^{j}-\rho^{\infty, j}\right)\left[Y_{j \mid S}\right]+\widehat{\Lambda}_{L \mid S} .
$$

We infer that $\theta_{L_{S}}$ contains a Kähler current, by the property 1.D.1, iii) and the fact that the above coefficients are positive. Its critical exponent is greater than 1 , by the definition of the set $I$ in (30), the proposition 1.D.1 and the fact that the restrictions $Y_{j \mid S}$ are smooth and they do not intersect each other. Moreover, the adjoint class $c_{1}\left(K_{S}\right)+\theta_{L_{S}}$ is pseudoeffective, by the compactness property of closed positive currents. Indeed, any weak limit of the currents $R_{\varepsilon}$ above will be positive, and the last term in the numerical identity (31) will converge to zero as $\varepsilon \rightarrow 0$.

Now we apply the induction hypothesis : the class $c_{1}\left(K_{S}\right)+\theta_{L_{S}}$ contains a non-zero, effective $\mathbb{R}$-divisor, which can be written as

$$
T_{S}:=\sum_{i \in K} \lambda^{i}\left[W_{i}\right]
$$

where $W_{i} \subset S$ are hypersurfaces. We consider now the current

$$
\widehat{T}_{S}:=\sum_{i \in K} \lambda^{i}\left[W_{i}\right]+\sum_{j \in J \backslash I} \rho^{\infty, j}\left[Y_{j \mid S}\right]+\sum_{j \in I} \nu^{j}\left[Y_{j \mid S}\right] ;
$$

from the relation (31) we get

$$
\widehat{T}_{S} \in c_{1}\left(K_{\widehat{X}}+S\right)+\theta_{\widehat{L} \mid S}
$$

During our discussion of the case where $\Theta_{K_{X}+L}$ has log poles and rational singularities, we have used the extension theorem [19] in order to lift the section whose zero 
set is (in the actual context) $\widehat{T}_{S}$. The obstructions in order to do the same thing now are the following :

(•) $\theta_{\widehat{L}}$ does not necessarily correspond to a $\mathbb{Q}$-bundle. In fact, we remark that this could happen even if at the very beginning it is the case for $\theta_{L}$-the reason is that the Lelong numbers of $\Theta_{K_{X}+L}$ are not rational numbers in general ;

(•) The current $\widehat{T}_{S}$ do not (necessarily) correspond to the zero set of a $\mathbb{Q}$-section ;

(•) The current $\Theta_{\varepsilon}$ is greater than $-\varepsilon \omega$, but not positive in general.

The way to deal with the first difficulty is by diophantine approximation, and will be explained in the next subsection. Then the second one is not a serious problem, since the method of Shokurov can be adapted to this setting. Finally, the slight negativity of the current $\Theta_{\varepsilon}$ has the following consequences : when we apply the "invariance of plurigenera" iteration, we can perform only a finite number of steps. However, we will show that as soon as the approximation is accurate enough, the number of steps is sufficiently large to allow us to conclude (here we use in an essential manner the strong positivity of $\widehat{\Lambda}_{L}$ ).

\section{$\S 1 . F$ Approximation}

In this paragraph we would like to prove the following diophantine approximation lemma (see [5], as well as [39] for similar considerations).

1.F.1 Lemma. For each $\eta>0$, there exist a positive integer $q_{\eta}$, a $\mathbb{Q}$-line bundle $\widehat{L}_{\eta}$ on $\widehat{X}$ and a closed positive current

$$
\widehat{T}_{S, \eta}:=\sum_{i \in K} \lambda_{\eta}^{i}\left[W_{i}\right]+\sum_{j \in J \backslash I} \rho_{\eta}^{\infty, j}\left[Y_{j \mid S}\right]+\sum_{j \in I} \nu_{\eta}^{j}\left[Y_{j \mid S}\right] ;
$$

such that:

A.1 The multiple $q_{\eta} \widehat{L}_{\eta}$ is a genuine line bundle, and the numbers

$$
\left(q_{\eta} \lambda_{\eta}^{i}\right)_{i \in K},\left(q_{\eta} \nu_{\eta}^{j}\right)_{j \in J},\left(q_{\eta} \rho_{\eta}^{\infty, j}\right)_{j \in J}
$$

are integers ;

A.2 We have $\widehat{T}_{S, \eta} \in\left\{K_{\widehat{X}}+S+\widehat{L}_{\eta \mid S}\right\}$;

A.3 We have $\left\|q_{\eta}\left(\widehat{L}-\widehat{L}_{\eta}\right)\right\|<\eta,\left|q_{\eta}\left(\lambda_{\eta}^{i}-\lambda^{i}\right)\right|<\eta$ and the analog relation for the $\left(\rho^{\infty, j}, \nu^{j}\right)_{j \in J}$ (here $\|\cdot\|$ denotes any norm on the real Neron-Severi space of $\left.\widehat{X}\right)$;

A.4 For each $\eta_{0}>0$, there exist a finite family $\left(\eta_{j}\right)$ such that $\left\{K_{\widehat{X}}+S\right\}+\theta_{\widehat{L}}$ belong to the convex hull of $\left\{K_{\widehat{X}}+S+\widehat{L}_{\eta_{j}}\right\}$ where $0<\eta_{j}<\eta_{0}$.

Proof (of the approximation lemma). We choose first an appropriate basis of the NeronSeveri space of $S$. 
Let $\alpha_{1}, \ldots, \alpha_{\rho}$ be a basis of the $\operatorname{NS}(X)$. The restrictions $\left(\alpha_{j \mid S}\right)$ generate a subspace of $\mathrm{NS}(S)$; let us assume that a basis of this subspace is given by $\left(\alpha_{j \mid S}\right)_{1 \leq j \leq r}$. We complete this free family with $\beta_{1}, \ldots, \beta_{\rho^{\prime}}$ to a basis of $\operatorname{NS}(S)$.

We compute the coordinates of the cohomology class of the current

$$
\widehat{T}_{S}=\sum_{i \in K} \lambda^{i}\left[W_{i}\right]+\sum_{j \in J \backslash I} \rho^{\infty, j}\left[Y_{j \mid S}\right]+\sum_{j \in I} \nu^{j}\left[Y_{j \mid S}\right] \in\left\{K_{\widehat{X}}+S\right\}+\theta_{\widehat{L} \mid S}
$$

with respect to the basis $(\alpha, \beta)$ above. Since $\left\{K_{\widehat{X}}+S\right\}+\theta_{\widehat{L}}$ is defined on $\widehat{X}$, there exist a set of real numbers $\left(b^{j}\right)$ such that

$$
\left\{K_{\widehat{X}}+S\right\}+\theta_{\widehat{L}}=\sum_{j=1}^{\rho} b^{j} \alpha_{j} .
$$

We equally have the rational numbers $\left(q_{p}^{j}\right)$ such that

$$
\alpha_{r+j \mid S}=\sum_{k=1}^{r} q_{j}^{k} \alpha_{k \mid S}
$$

by our assumption, and therefore we obtain

$$
\left\{K_{\widehat{X}}+S\right\}+\theta_{\widehat{L} \mid S}=\sum_{p=1}^{r}\left(b^{p}+\sum_{j=1}^{\rho-r} b^{r+j} q_{j}^{p}\right) \alpha_{p \mid S} .
$$

In order to simplify the writing, we denote by $a^{p}:=b^{p}+\sum_{j=1}^{\rho-r} b^{r+j} q_{j}^{p}$.

Next, we are going to express the coordinates of $c_{1}\left(W_{j}\right)$ and $c_{1}\left(Y_{j}\right)$ with respect to the basis $(\alpha, \beta)$. There exist the rational numbers $\left(x_{j}^{p}\right),\left(y_{j}^{q}\right),\left(z_{j}^{k}\right)$ such that

$$
W_{j} \equiv \sum_{p=1}^{r} x_{j}^{p} \alpha_{p \mid S}+\sum_{p=1}^{\rho^{\prime}} y_{j}^{p} \beta_{p}
$$

as well as

$$
\begin{aligned}
Y_{j \mid S} & \equiv \sum_{k=1}^{\rho} z_{j}^{k} \alpha_{k \mid S} \\
& \equiv \sum_{k=1}^{r}\left(z_{j}^{k}+\sum_{p=1}^{\rho-r} z_{j}^{p+r} q_{p}^{k}\right) \alpha_{k \mid S}
\end{aligned}
$$

By the relations (32), (33) and (36) we get the next equalities

$$
\sum_{i \in K} \lambda^{i} x_{i}^{k}+\sum_{j \in J \backslash I} \rho^{\infty, j}\left(z_{j}^{k}+\sum_{p=1}^{\rho-r} z_{j}^{p+r} q_{p}^{k}\right)+\sum_{j \in I} \nu^{j}\left(z_{j}^{k}+\sum_{p=1}^{\rho-r} z_{j}^{p+r} q_{p}^{k}\right)=a^{k}
$$


for $p=1, \ldots, \rho^{\prime}$ and $k=1, \ldots, r$.

Let $Z$ be the matrix whose coefficients are the rational numbers $\left(x_{j}^{p}\right),\left(y_{j}^{q}\right)$ as well as $\left(z_{j}^{k}+\sum_{p=1}^{\rho-r} z_{j}^{p+r} q_{p}^{k}\right)$ such that the above equations can be written as

$$
Z \Lambda=V
$$

where $V$ is the vector whose first $\rho^{\prime}$ coefficients are zero, and the next ones are just the $\left(a^{k}\right)$. Conversely, if we have a solution $(\lambda, \rho, \nu)$ of the linear system (39) corresponding to a vector $V$ whose first $\rho^{\prime}$ components are zero, then the cohomology class of the corresponding $\widehat{T}_{S}$ belong to the space generated by $\left(\alpha_{j}\right)$.

We denote by $H \subset \mathrm{NS}(S)$ the vector space obtained by intersecting the image of $Z$ with the space defined by the vanishing of the first $\rho^{\prime}$ coordinates ; the equality (39) show that the vector $V$ belong to $H$.

Next, the main fact is that the subspace $H$ is defined over the rational numbers i.e. there exist a basis $h_{1}, \ldots h_{l}$ of $H$ which can be expressed in $(\alpha, \beta)$ with rational coefficients (this is a consequence of the fact that the matrix $Z$ has rational coefficients). Then we write our vector $V$ with respect to this basis of $H$, as follows

$$
V=\sum_{p=1}^{l} c^{p} h_{p}
$$

We will use the next fact from the "diophantine approximation" theory.

1.F.2 Fact. Let $x^{1}, \ldots, x^{s}$ be a finite set of real numbers; there exist a constant $C>0$ such that for any $\eta>0$, there exist $q_{\eta} \in \mathbb{Z}_{+}$such that :

(1) For each $j=1, \ldots, s$, there exist integers $p^{j}$ so that we have $\left|q_{\eta} x^{j}-p^{j}\right|<C \eta$ we denote by $X$ the vector in $\mathbb{R}^{s}$ whose coefficients are the $\left(x^{j}\right)$, and by $X_{\eta}$ the vector given by the rational approximations $\frac{p^{j}}{q_{\eta}}$;

(2) Given a positive real $\eta_{0}$, there exist a family of approximations $X_{\eta_{j}}$ as above, with $\eta_{j}<\eta_{0}$ such that the vector $X$ belongs to the convex hull of $X_{\eta_{j}}$.

Proof. Indeed, we show along the next lines that the above statement is a consequence of the Kronecker theorem (see e.g. Hardy-Wright, [21]).

We consider the maximal family, say $x^{1}, \ldots, x^{p}$ such that the numbers $1, x^{1}, \ldots, x^{p}$ are independent over $\mathbb{Q}$. The integer $p$ above can be assumed to be greater than 1 , because if not all $\left(x^{j}\right)$ are rational, and in this case the above result is obvious. Thus, we have

$$
x^{p+j}=\sum_{k=1}^{p} r_{k}^{j} x^{k}+r_{0}^{j}
$$

for all $j=1, \ldots, s-p$, where $\left(r_{k}^{j}\right)$ is a set of rational numbers. Let $A=\left(r_{k}^{j}\right)$ and $B=\left(r_{0}^{j}\right)$; then our vector $\left(x^{1}, \ldots, x^{s}\right)$ become an a point on the graph of the affine map

$$
Y \rightarrow A Y+B
$$


defined on $\mathbb{R}^{p} \rightarrow \mathbb{R}^{s-p}$.

Let $\eta>0$ be a positive rational number. The theorem of Kronecker (see [21]) imply that the set $\left(\left\{\varepsilon_{1} q x^{1}\right\}, \ldots,\left\{\varepsilon_{p} q x^{p}\right\}\right)_{q \in \mathbb{Z}_{+}}$is dense in the cube $[0,1]^{p}$, for any choice of the quantities $\varepsilon_{j}$ within the set $\{-1,1\}$. Here we use the fact that if the family $1, x^{1}, \ldots, x^{p}$ is independent over $\mathbb{Q}$, then so is $1, \varepsilon_{1} x^{1}, \ldots, \varepsilon_{p} x^{p}$, for any choice of $\left(\varepsilon_{j}\right)$ as above.

We consider the vectors

$$
X_{\varepsilon, q}:=\left(x^{1}-\frac{\varepsilon_{1}}{q}\left\{\varepsilon_{1} q x^{1}\right\}, \ldots, x^{p}-\frac{\varepsilon_{p}}{q}\left\{\varepsilon_{p} q x^{p}\right\}\right) ;
$$

our first remark is that they have rational coefficients, as one can easily see. Next, for any $\left(\varepsilon_{j}\right)$ as above, we will consider the vectors $X_{\varepsilon, q}$ such that $\left\{\varepsilon_{j} q x^{j}\right\}<\eta$, for any $j=1, \ldots, p$. Finally, we can select a finite family from the $\left(X_{\varepsilon, q}\right)$ above such that the vector $\left(x^{1}, \ldots, x^{p}\right)$ is in the interior of the polygon they define.

But then, the vector $(X, A X+B)$ is in the convex hull of the

$$
\left(X_{\varepsilon, q}, A X_{\varepsilon, q}+B\right)
$$

and this completes the proof of 1.F.1.

We apply now the diophantine approximation statement above for the following set of real numbers :

$$
\left(c^{j}\right)_{1 \leq j \leq l},\left(b^{r+j}\right)_{1 \leq j \leq \rho-r},\left(\rho^{\infty, j}\right)_{j \in J},\left(\nu^{j}\right)_{j \in J},\left(\lambda^{j}\right)_{j \in K} .
$$

To complete the proof of the lemma, will trace our steps back in order to obtain the approximations of the $\mathbb{R}$-sections/bundles we are interested in.

We first define

$$
V_{\eta}:=\sum_{j} c_{\eta}^{j} h_{j} \in H
$$

and its coefficients with respect to the basis $(\alpha, \beta)$ will define the rational approximations $\left(a_{\eta}^{j}\right)$ of the coefficients $\left(a^{j}\right)$ above ; moreover, the coefficients corresponding to $\left(\beta_{l}\right)$ are zero. Then we set

$$
b_{\eta}^{j}:=a_{\eta}^{j}-\sum_{k=1}^{\rho-r} b_{\eta}^{r+k} q_{k}^{j}
$$

and

$$
F_{\eta}: \equiv \sum_{j=1}^{\rho} b_{\eta}^{j} \alpha_{j},
$$

that is to say we consider any $\mathbb{Q}$-line bundle whose Chern class is given by the above expression. Finally, we can choose the vector $\Lambda_{\eta}$ such that

$$
Z \Lambda_{\eta}=V_{\eta}
$$

with the property that

$$
\left\|q_{\eta}\left(\Lambda_{\eta}-\Lambda\right)\right\|<C \eta
$$


and such that $m_{0} q_{\eta} \Lambda_{\eta}$ has all the components integral numbers, where $m_{0}$ is a fixed positive integer. Such a vector can indeed be found, by the usual theory of linear systems ; the constant $m_{0}$ is due to the inverse of some square matrix extracted from $Z$. It is at this point that we need to have at hand the approximations of some of the $\left(\lambda^{j}\right),\left(\rho^{\infty, j}\right),\left(\nu^{j}\right)$; the others will be imposed by the linear system $(40)$.

We recall that all the components of the vector $\Lambda$ are positive real numbers, therefore the approximation $\Lambda_{\eta}$ will have the same property, if $\eta \ll 1$.

The approximation $\widehat{T}_{S, \eta}$ of the the current $\widehat{T}_{S}$ in our lemma 1.F.1 is obtained simply by plugging in the coefficients of $\Lambda_{\eta}$.

The equation (40) show that

$$
\left\{\widehat{T}_{S, \eta}\right\}=c_{1}\left(F_{\eta \mid S}\right)
$$

moreover, the relation (1) in 1.F.2 imply

$$
\left\|q_{\eta}\left(\left\{K_{\widehat{X}}+S\right\}+\theta_{\widehat{L}}-\left\{F_{\eta}\right\}\right)\right\|<C \eta
$$

moreover, we can assume that $q_{\eta} F_{\eta}$ is integral (here we are a bit sloppy, since some additional denominators can occur because of the coefficients $(x, y, q)$ above, but they are fixed independently of $\eta$, so we just ignore them).

Finally, we remark that $\left\{K_{\widehat{X}}+S\right\}+\theta_{\widehat{L}}$ belong to the convex hull of the $F_{\eta}$ thanks to the second part of 1.F.2. This is indeed the case, since the vector $\left(b_{1}, \ldots, b_{\rho}\right)$ is the image of $\left(c_{1}, \ldots, c_{l}, b_{r+1}, \ldots, b_{\rho}\right)$ via a linear map, and we use precisely the same map (whose associated matrix with respect to the basis above is has rational coefficients) to define the approximations.

We define $\widehat{L}_{\eta}$ such that

$$
K_{\widehat{X}}+S+\widehat{L}_{\eta} \equiv F_{\eta}
$$

and the proof of the lemma is finished.

\section{§1.G The method of Shokurov}

Our concern in this paragraph will be to "convert" the current $\widehat{T}_{S, \eta}$ into a genuine section $s_{\eta}$ of the bundle $q_{\eta}\left(K_{\widehat{X}}+S+\widehat{L}_{\eta}\right)$. To this end, we will apply a classical trick of Shokurov, in the version revisited by Siu in his recent work [39]. A crucial point is that by a careful choice of the metrics we use, the $L^{2}$ estimates will allow us to have a very precise information concerning the vanishing of $s_{\eta}$.

1.G.1 Proposition. There exist a section

$$
s_{\eta} \in H^{0}\left(S, q_{\eta}\left(K_{S}+\widehat{L}_{\eta \mid S}\right)\right)
$$

whose zero set contains the divisor

$$
q_{\eta}\left(\sum_{j \in J \backslash I} \rho_{\eta}^{\infty, j}\left[Y_{j \mid S}\right]+\sum_{j \in I} \nu_{\eta}^{j}\left[Y_{j \mid S}\right]\right)
$$


for all $0<\eta \ll 1$.

Proof(of the proposition). We express the bundle we are interested in as an adjoint bundle as follows

$$
q_{\eta}\left(K_{S}+\widehat{L}_{\eta \mid S}\right)=K_{S}+\left(q_{\eta}-1\right)\left(K_{S}+\widehat{L}_{\eta \mid S}\right)+\widehat{L}_{\eta \mid S}
$$

In order to use the classical vanishing theorems, we have to endow the bundle

$$
\left(q_{\eta}-1\right)\left(K_{S}+\widehat{L}_{\eta \mid S}\right)+\widehat{L}_{\eta \mid S}
$$

with an appropriate metric. We first consider first the $\mathbb{Q}$-bundle $\widehat{L}_{\eta}$; we will construct a metric on it from the decomposition

$$
c_{1}\left(\widehat{L}_{\eta}\right)=\theta_{\widehat{L}}+\left(c_{1}\left(\widehat{L}_{\eta}\right)-\theta_{\widehat{L}}\right)
$$

The second term above admits a smooth representative whose local weights are bounded by $\frac{\eta}{q_{\eta}}$ in $\mathcal{C}^{\infty}$ norm, by the approximation relation $A .3$. As for the first one, we recall that the class $\theta_{\widehat{L}}$ contains the representative

$$
\sum_{j \in J} \nu^{j} Y_{j}+\widehat{\Lambda}_{L}
$$

where the $(1,1)$-form $\widehat{\Lambda}_{L}$ has the positivity properties in 1.D.1, iii).

Now, the first metric we consider on $\widehat{L}_{\eta \mid S}$ is given by the perturbation of the current (41) as follows :

$$
\sum_{j \in I} \max \left(\nu^{j}, \nu_{\eta}^{j}\right) Y_{j \mid S}+\sum_{j \in J \backslash I} \nu^{j} Y_{j \mid S}+\widehat{\Lambda}_{L \mid S}+\Theta(\eta)_{\mid S}
$$

where $\Theta(\eta)$ is a non-singular $(1,1)$-form on $\widehat{X}$ in the class of the current

$$
\sum_{j \in I}\left(\nu^{(j)}-\max \left(\nu^{(j)}, \nu_{\eta}^{(j)}\right)\right)\left[Y_{j}\right]
$$

plus $c_{1}\left(\widehat{L}_{\eta}\right)-\theta_{\widehat{L}} ;$ we can assume that it is greater than $-C \frac{\eta}{q_{\eta}}$.

The smooth term $\widehat{\Lambda}_{L \mid S}$ is semi-positive on $S$ and strictly positive at the generic point ; in order to gain the strict positivity needed in the vanishing theorems, we recall the following standard result (see e.g. [15], [28]).

Fact. There exist a Kähler metric $\Omega$ on $S$, a positive constant $C$ and a family of currents $\widehat{\Lambda}_{L, S}^{\varepsilon} \in\left\{\widehat{\Lambda}_{L \mid S}\right\}$ such that :

(1) We have $\widehat{\Lambda}_{L, S}^{\varepsilon} \geq \varepsilon \Omega$;

(2) The Lelong number of $\widehat{\Lambda}_{L, S}^{\varepsilon}$ at each point of the manifold is smaller than $\varepsilon C$. 
Thus, for any $\eta>0$, we can define an $\varepsilon$ such that the corresponding current $\widehat{\Lambda}_{L, S}^{\varepsilon}$ verify the next properties.

$M_{1}$ ) The current $\widehat{\Lambda}_{L, S}^{\varepsilon}+\Theta(\eta)$ dominates a small multiple of $\Omega$, which depends on $\eta$, but fortunately this does not matter for the purposes of this paragraph ;

$M_{2}$ ) The Lelong number of $\widehat{\Lambda}_{L, S}^{\varepsilon}$ at each point of $S$ is smaller than $C \frac{\eta}{q_{\eta}}$.

In conclusion, we can define a metric on $\widehat{L}_{\eta \mid S}$ with the following curvature current

$$
\sum_{j \in I} \max \left(\nu^{j}, \nu_{\eta}^{j}\right) Y_{j \mid S}+\sum_{j \in J \backslash I} \nu^{j} Y_{j \mid S}+\widehat{\Lambda}_{L, S}^{\varepsilon}+\Theta(\eta)_{\mid S}
$$

we remark that it is a Kähler current, and its critical exponent is strictly greater than 1 , provided that $\eta \ll 1$.

Next, we define a singular metric on the bundle $\left(q_{\eta}-1\right)\left(K_{S}+\widehat{L}_{\eta \mid S}\right)$ whose curvature form is equal to $\left(q_{\eta}-1\right) \widehat{T}_{S, \eta}$ and we denote by $h_{\eta}$ the resulting metric on the bundle

$$
\left(q_{\eta}-1\right)\left(K_{S}+\widehat{L}_{\eta \mid S}\right)+L_{\eta \mid S} .
$$

The current $q_{\eta} \widehat{T}_{S, \eta}$ corresponds to the current of integration along the zero set of the section $u_{\eta}$ of the bundle

$$
q_{\eta}\left(K_{S}+\widehat{L}_{\eta \mid S}\right)+\rho
$$

where $\rho$ is a topologically trivial line bundle on $S$.

By the Kawamata-Viehweg-Nadel vanishing theorem we have

$$
H^{j}\left(S, q_{\eta}\left(K_{S}+\widehat{L}_{\eta}\right) \otimes \mathcal{J}\left(h_{\eta}\right)\right)=0
$$

for all $j \geq 1$, and the same is true for the bundle $q_{\eta}\left(K_{S}+\widehat{L}_{\eta}\right)+\rho$, since $\rho$ carries a metric with zero curvature. Moreover, the section $u_{\eta}$ belong to the multiplier ideal of the metric $h_{\eta}$ above, as soon as $\eta$ is small enough, because the multiplier ideal of the metric (43) on the bundle $\widehat{L}_{\eta \mid S}$ will be trivial. Since the Euler characteristic of the two bundles is the same, we infer that

$$
H^{0}\left(S, q_{\eta}\left(K_{S}+\widehat{L}_{\eta}\right) \otimes \mathcal{J}\left(h_{\eta}\right)\right) \neq 0
$$

We denote by $s_{\eta}$ any non-zero element in the group above ; we show now that its zero set satisfy the requirements in the lemma. Indeed, locally at any point of $x \in S$ we have

$$
\int_{(S, x)} \frac{\left|f_{s}\right|^{2}}{\prod_{j \in J \backslash I}\left|f_{j}\right|^{2 \rho_{\eta}^{\infty, j}\left(q_{\eta}-1\right)+2 \widetilde{\nu}_{\eta}^{(j)}} \prod_{j \in I}\left|f_{j}\right|^{2 \nu_{\eta}^{j}\left(q_{\eta}-1\right)+2 \widetilde{\nu}_{\eta}^{j}}} d \lambda<\infty
$$

where $\widetilde{\nu}_{\eta}^{j}:=\nu^{j}$ if $j \in J \backslash I$ and $\widetilde{\nu}_{\eta}^{j}:=\max \left\{\nu_{\eta}^{j}, \nu^{j}\right\}$ if $j \in I$; we denote by $f_{s}$ the local expression of the section $s$, and we denote by $f_{j}$ the local equation of $Y_{j} \cap S$. 
But the we have

$$
\int_{(S, x)} \frac{\left|f_{s}\right|^{2}}{\prod_{j \in J \backslash I}\left|f_{j}\right|^{2 \rho_{\eta}^{\infty, j} q_{\eta}} \prod_{j \in I}\left|f_{j}\right|^{2 \nu_{\eta}^{j} q_{\eta}}} d \lambda<\infty
$$

for all $\eta \ll 1$ (by the definition of the set $I$ and the construction of the metric on $\widehat{L}_{\eta \mid S}$ ). Therefore, the lemma is proved.

\section{$\S 1 . H$ The method of Siu and Hacon-McKernan}

We have arrived now at the last step in our proof : for all $0<\eta \ll 1$, the section $s_{\eta}$ admit an extension on $\widehat{X}$. Once this is done, we just use the point A.4 of the approximation lemma 1.F.1, in order to infer the existence of a $\mathbb{R}$-section of the bundle $K_{\widehat{X}}+S+\widehat{L}$, and then the relation 1.D.1, iv) to conclude.

In order to explain our approach in the simplest possible way, we consider next the approximation of the usual setup of the extension of twisted pluricanonical sections ; afterwards we will compare it with our current situation. We will use different notations, to avoid any confusion that may occur.

Let $\bar{X}$ be a projective, non-singular manifold ; let

$$
\mathcal{S}:=\left\{\bar{S},\left(\bar{Y}_{j}\right)_{j \in J}, \bar{L}, \bar{A}, T\right\}
$$

be a set of objects on $\bar{X}$, with the following properties.

$\left(\mathbf{U}_{\mathbf{0}}\right) \bar{S}$ and $\left(\bar{Y}_{j}\right)_{j \in J}$ are non-singular hypersurfaces of $\bar{X}$, with normal crossings and such that $\forall j \neq k$ we have $\bar{Y}_{j} \cap \bar{Y}_{k}=\emptyset$;

$\left(\mathbf{U}_{\mathbf{1}}\right) \bar{L}$ is a $\mathbb{Q}$-line bundle, which admits the following numerical decomposition

$$
\bar{L} \equiv \bar{\Delta}_{1}+\bar{\Delta}_{2}
$$

where

$$
\bar{\Delta}_{1}=\sum_{j \in J \backslash I} \alpha^{j}\left[\bar{Y}_{j}\right]
$$

and

$$
\bar{\Delta}_{2}=\sum_{j \in I} \alpha^{j}\left[\bar{Y}_{j}\right]+\bar{\Lambda}_{2}
$$

The numbers $\alpha^{j}$ above are rational and moreover $\left.\alpha^{j} \in\right] 0,1\left[\right.$; also, $\bar{\Lambda}_{2}$ is a Kähler current, with logarithmic poles and rational cohomology class, whose generic Lelong number along $\bar{S}$ is equal to zero, i.e.

$$
\nu_{\bar{S}}\left(\bar{\Lambda}_{2}\right)=0
$$

In this case the restriction $\bar{\Lambda}_{2 \mid \bar{S}}$ is well defined and we denote by

$$
\nu:=\sup _{x \in \bar{S}} \nu\left(\bar{\Lambda}_{2 \mid \bar{S}}, x\right)
$$


$\left(\mathbf{U}_{2}\right) T$ is a closed current with logarithmic poles in the cohomology class of the bundle $K_{\bar{X}}+\bar{S}+\bar{L}$ and $\bar{A}$ is an ample line bundle such that :

( $\left.\mathbf{U}_{\mathbf{2 . 1}}\right)$ We have

$$
T \geq-C_{T} \Theta(A)
$$

where $C_{T}$ is a positive constant, and $\Theta(A)$ is a positive and non-singular curvature form corresponding to a metric on $A$.

( $\left.\mathbf{U}_{\mathbf{2 . 2}}\right)$ The restriction of $T$ to $\bar{S}$ is well-defined and we have

$$
T_{\mid \bar{S}}:=\sum_{j \in J} \bar{\theta}^{j}\left[\bar{Y}_{j \mid \bar{S}}\right]+R
$$

where $\bar{\theta}^{j}$ are positive real numbers, and $R$ is a closed current on $\bar{S}$, whose generic Lelong numbers along $\bar{Y}_{j \mid \bar{S}}$ is equal to zero ;

$\left(\mathbf{U}_{3}\right)$ Let $q \in \mathbb{Z}_{+}$be a positive integer, such that $q \bar{L}$ and $q \alpha^{j}$ are integral, for any $j \in J$, and such that

$$
q \nu<1
$$

that is to say, the multiple $q$ is not allowed to be greater than the inverse of the maximum of the singularities of $\bar{\Lambda}_{2 \mid S}$. We assume that there exist a section

$$
s \in H^{0}\left(\bar{S}, q\left(K_{\bar{S}}+\bar{L}\right)\right)
$$

whose zero set contains the divisor

$$
q\left(\sum_{j \in J \backslash I} \rho^{j}\left[\bar{Y}_{j \mid \bar{S}}\right]+\sum_{j \in I} \alpha^{j}\left[\bar{Y}_{j \mid \bar{S}}\right]\right)
$$

such that

$$
\rho^{j} \geq \theta^{j}-C_{T}^{\prime}
$$

for each $j \in J \backslash I$; in the above relation, $C_{T}^{\prime}$ is a positive constant.

Since the coefficients of $q \bar{\Delta}_{1}$ are positive integers strictly less than $q$, we have the decomposition (see [4], [18])

$$
q \bar{\Delta}_{1}=L_{1}+\ldots+L_{q-1}
$$

such that for each $m=1, \ldots, q-1$, we have

$$
L_{m}:=\sum_{j \in I_{m} \subset J \backslash I} \bar{Y}_{j}
$$


We denote by $L_{q}:=q \bar{\Delta}_{2}$ and

$$
L^{(p)}:=p\left(K_{\bar{X}}+\bar{S}\right)+L_{1}+\ldots+L_{p}
$$

where $p=1, \ldots, q$. By convention, $L^{(0)}$ is the trivial bundle.

$\left(\mathbf{U}_{4}\right)$ The ample bundle $A$ is assumed to be positive enough such that the next conditions hold.

$(\dagger)$ For each $0 \leq p \leq q-1$, the bundle $L^{(p)}+q A$ is generated by its global sections, which we denote by $\left(s_{j}^{(p)}\right)$.

$\left(\dagger^{2}\right)$ Any section of the bundle $L^{(q)}+q A_{\mid S}$ admits an extension to $\bar{X}$.

$\left(\dagger^{3}\right)$ We endow the bundle corresponding to $\left(\bar{Y}_{j}\right)_{j \in J}$ and $K_{\bar{X}}+\bar{S}$ with non-singular metrics, and we denote by $\widetilde{\varphi}_{m}$ the induced metric on $L_{m}$. Then for each $m=1, \ldots, q$, the functions

$$
\widetilde{\varphi}_{L_{m}}+1 / 3 \varphi_{A} \text { and } \widetilde{\varphi}_{K}+1 / 3 \varphi_{A}
$$

are strictly psh, where $\widetilde{\varphi}_{K}$ is the non-singular metric on the bundle $K_{\bar{X}}+\bar{S}$.

Under the numerous assumptions/normalizations above, we formulate the next statement.

Claim. The section

$$
s^{\otimes k} \otimes s_{j}^{(p)} \in H^{0}\left(\bar{S}, L^{(p)}+k L^{(q)}+q A_{\mid \bar{S}}\right)
$$

extend to $\bar{X}$, for each $p=0, \ldots, q-1, j=1, \ldots, N_{p}$ and $k \in \mathbb{Z}_{+}$such that

$$
k \max \left(C_{T}, C_{T}^{\prime}\right) \leq 1 / 4 \text {. }
$$

As one can see, the main differences between the present situation and the usual version of the invariance of plurigenera (see e.g. [9], [18], [19], [24], [26], [33], [37], [38], [41], [42], [43], [44]) is visible in the relations $\mathcal{R}_{T}, \mathcal{R}_{\nu}$ and $\mathcal{R}_{s}$. Indeed, the "standard" assumptions in the articles quoted above are as follows.

(•) The current $T$ in $\left(\mathbf{U}_{\mathbf{2}}\right)$ is positive, that it to say $C_{T}=0$;

(•) The current $\bar{\Lambda}_{2}$ in $\left(\mathbf{U}_{\mathbf{1}}\right)$ is non-singular ;

(•) The restriction $T_{\mid \bar{S}}$ and the support of $\bar{\Delta}_{1}$ have no common components and therefore we can take $C_{T}^{\prime}=0$.

Under this perspective, the statement above can be seen as a natural generalization of the usual setting ; in substance, we are about to say that the more general hypothesis we are forced to consider induce an effective limitation of the number of iterations we are allowed to perform.

The rest of the present paragraph is organized as follows. We start with the proof of the claim above in 1.H.A. In the paragraph 1.H.B we show that the family of bundles $\left(\widehat{L}_{\eta}\right)$ is "uniform", in the sense that we have a precise control of the corresponding constants $C_{T}, C_{T}^{\prime}$ as $\eta \rightarrow 0$. We complete the proof of the non-vanishing theorem in 1.H.C, by showing that the claim imply the extension of $\left(s_{\eta}\right)$. 


\section{$\S 1 . H . A$ Proof of the claim}

We will review here the main steps of the arguments in the usual invariance of plurigenera ; to start with, we recall the following very useful integrability criteria (see e.g. [4]).

1.H.A.1 Lemma([4]). Let $\Theta$ be a (1,1)-current with logarithmic poles on a manifold $\bar{S}$, such that $\Theta \geq-C \omega$, where $C$ is a positive real, and $\omega$ is a metric on $\bar{S}$. We consider equally the non-singular hypersurfaces $\bar{Y}_{j} \subset \bar{S}$ for $j=1, \ldots, N$ such that $\bar{Y}_{j} \cap \bar{Y}_{i}=\emptyset$ if $i \neq j$, and such that the generic Lelong number of $\Theta$ along each of the $\bar{Y}_{j}$ is zero. Then there exist a constant $\varepsilon_{0}:=\varepsilon_{0}(\{\Theta\}, C)$ depending only on the cohomology class of the current $\Theta$ and its lower bound such that for all positive real numbers $\delta \in] 0,1]$ and $\varepsilon \leq \varepsilon_{0}$ we have

$$
\int_{\bar{S}} \exp \left(-(1-\delta) \sum_{j} \varphi_{\bar{Y}_{j}}-\varepsilon \varphi_{\Theta}\right) d \lambda<\infty
$$

In the statement above, we denote by $\varphi_{\bar{Y}_{j}}$ the potential of the current $\left[\bar{Y}_{j}\right]$. We remark that strictly speaking the quantity under the sum above is not global, but we are only interested in the singularities of the objects above.

We will equally need the following version of the Ohsawa-Takegoshi theorem, obtained by McNeal-Varolin in [29] (see also [2], [13], [32], [38]) ; it will be our main technical tool in the proof of the claim.

1.H.A.2 Theorem ([29]). Let $X$ be a projective $n$-dimensional manifold, and let $S \subset X$ be a non-singular hypersurface. Let $F$ be a line bundle, endowed with a metric $h_{F}$. We assume the existence of some non-singular metric $h_{S}$ on the bundle $\mathcal{O}(\mathcal{S})$ such that :

(1) $\frac{\sqrt{-1}}{2 \pi} \Theta_{F} \geq 0$ on $X$;

(2) $\frac{\sqrt{-1}}{2 \pi} \Theta_{F}-\alpha \frac{\sqrt{-1}}{2 \pi} \Theta_{S} \geq 0$ for some $\alpha>0$;

(3) The restriction of the metric $h_{F}$ on $S$ is well defined.

Then every section $u \in H^{0}\left(S,\left(K_{X}+S+F_{\mid S}\right) \otimes \mathcal{I}\left(h_{F \mid S}\right)\right)$ admits an extension $U$ to $X$ such that

$$
c_{n} \int_{X} U \wedge \bar{U} \exp \left(-\varphi_{F}-\varphi_{S}-\log \left(|s|^{2} \log ^{2}(|s|)\right)\right)<\infty
$$

where $s$ is a section whose zero set is precisely the hypersurface $S$ and its norm in the integral above is measured with respect to $h_{S}$.

We will use inductively the extension theorem 1.H.A.2, in order to derive a lower bound for the power $k$ we can afford in the invariance of plurigenera algorithm, under the conditions $\left(\mathbf{U}_{\mathbf{j}}\right)_{1 \leq \mathbf{j} \leq 4}$; the first steps are as follows. 
(1) For each $j=1, \ldots, N_{0}$, the section $s \otimes s_{j}^{(0)} \in H^{0}\left(\bar{S}, L^{(q)}+q A_{\mid \bar{S}}\right)$ admits an extension $U_{j}^{(q)} \in H^{0}\left(\bar{X}, L^{(q)}+q A\right)$, by the property $\left(\mathbf{U}_{4}\right), \dagger \dagger$.

(2) We use the sections $\left(U_{j}^{(q)}\right)$ to construct a metric $\varphi^{(q)}$ on the bundle $L^{(q)}+q A$.

(3) Let us consider the section $s \otimes s_{j}^{(1)} \in H^{0}\left(\bar{S}, L^{(1)}+L^{(q)}+q A_{\mid \bar{S}}\right)$. We remark that the bundle

$$
L^{(1)}+L^{(q)}+q A=K_{\bar{X}}+\bar{S}+L_{1}+L^{(q)}+q A
$$

can be written as $K_{\bar{X}}+\bar{S}+F$ where

$$
F:=L_{1}+L^{(q)}+q A
$$

thus we have to construct a metric on $F$ which satisfy the curvature and integrability assumptions in the Ohsawa-Takegoshi-type theorem above.

Let $\delta, \varepsilon$ be positive real numbers ; we endow the bundle $F$ with the metric given by

$$
\varphi_{\delta, \varepsilon}^{(q)}:=(1-\delta) \varphi_{L_{1}}+\delta \widetilde{\varphi}_{L_{1}}+(1-\varepsilon) \varphi^{(q)}+\varepsilon q\left(\varphi_{A}+\varphi_{T}\right)
$$

where the metric $\widetilde{\varphi}_{L_{1}}$ is smooth (no curvature requirements) and $\varphi_{L_{1}}$ is the singular metric induced by the divisors $\left(Y_{j}\right)_{j \in I_{1}}$, see $\left(\mathbf{U}_{\mathbf{4}}\right),\left(\dagger^{3}\right)$. if

We remark that the curvature conditions in the extension theorem will be fulfilled

$$
\delta<\varepsilon q
$$

since we are interested in the integers $k$ such that

$$
k \max \left(C_{T}, C_{T}^{\prime}\right) \leq 1 / 4,
$$

thus implicitly $C_{T}<1 / 3$ and the negativity of the curvature induced by the term $\delta \widetilde{\varphi}_{L_{1}}$ will be absorbed by $A$. We use here the relations in $\left(\mathbf{U}_{4}\right),\left(\dagger^{3}\right)$ and $\left(\dagger^{4}\right)$.

Next we claim that the sections $s \otimes s_{j}^{(1)}$ are integrable with respect to the metric defined in (44), provided that the parameters $\varepsilon, \delta$ are chosen in an appropriate manner. Indeed, we have to prove that

$$
\int_{\bar{S}} \frac{\left|s \otimes s_{j}^{(1)}\right|^{2}}{\left(\sum_{r}\left|s \otimes s_{r}^{(0)}\right|^{2}\right)^{1-\varepsilon}} \exp \left(-(1-\delta) \varphi_{L_{1}}-\varepsilon q \varphi_{T}\right) d V<\infty
$$

since the sections $\left(s_{r}^{(0)}\right)$ have no common zeroes, it is enough to show that

$$
\int_{\bar{S}}|s|^{2 \varepsilon} \exp \left(-(1-\delta) \varphi_{L_{1}}-\varepsilon q \varphi_{T}\right) d V<\infty
$$

(we have abusively removed the smooth weights in the above expressions, to simplify the writing).

Now the property $\left(\mathbf{U}_{\mathbf{3}}\right)$ concerning the zero set of $s$ is used : the above integral is convergent, provided that we have

$$
\int_{\bar{S}} \exp \left(-(1-\delta) \varphi_{L_{1}}-\varepsilon q\left(\varphi_{T}-\sum_{j \in J \backslash I} \rho^{j} \varphi_{Y_{j}}-\sum_{j \in I} \alpha^{j} \varphi_{Y_{j}}\right)\right) d V<\infty .
$$


We remark that we have

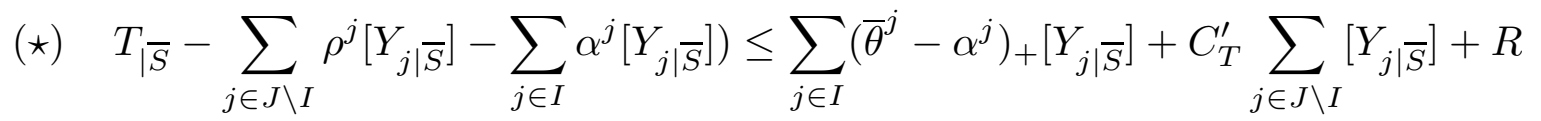

(by the property $\left(\mathbf{U}_{\mathbf{3}}\right)$ ) and therefore, we have an explicit measure of the size of the common part of the 1-codimensional components of the difference current above and those of $L_{1}$. We use the notation

$$
x_{+}:=\max (x, 0)
$$

in the previous expression.

We check next the conditions on $\varepsilon, \delta$ in order to insure the hypothesis of 1.H.A.1. The cohomology class of the current

$$
\sum_{j \in I}\left(\bar{\theta}^{j}-\alpha^{j}\right)_{+}\left[Y_{j \mid \bar{S}}\right]+R
$$

together with 1.H.C.1 will provide us with a quantity which we denote by $\varepsilon_{0}$ (in the context we are interested in, it can even be assumed to be independent on $\eta$, but this does not matter). The singular part corresponding to $j \in J \backslash I$ in the expression ( $\star$ ) will be incorporated into the $(1-\delta) \varphi_{L_{1}}$ therefore we impose the relation

$$
1-\delta+q \varepsilon C_{T}^{\prime}<1
$$

In conclusion, the positivity and integrability conditions will be satisfied provided that

$$
q \varepsilon C_{T}^{\prime}<\delta<\varepsilon q \leq \varepsilon_{0}
$$

We can clearly choose the parameters $\delta, \varepsilon$ in order to satisfy (45), again by the implicit assumption in the claim.

(4) We apply the extension theorem and we get $U_{j}^{(q+1)}$, whose restriction on $\bar{S}$ is precisely $s \otimes s_{j}^{(1)}$.

The claim will be obtained by iterating the procedure (1)-(4) several times, and estimating carefully the influence of the negativity of $T$ on this process. Indeed, assume that we already have the set of global sections

$$
U_{j}^{(k q+p)} \in H^{0}\left(\bar{X}, L^{(p)}+k L^{(q)}+q A\right)
$$

which extend $s^{\otimes k} \otimes s_{j}^{(p)}$. They induce a metric on the above bundle, denoted by $\varphi^{(k q+p)}$. If $p<q-1$, then we define the family of sections

$$
s^{\otimes k} \otimes s_{j}^{(p+1)} \in H^{0}\left(\bar{S}, L^{(p+1)}+k L^{(q)}+q A_{\mid \bar{S}}\right)
$$


on $\bar{S}$. As in the step (3) above we remark that we have

$$
L^{(p+1)}=K_{\bar{X}}+\bar{S}+L_{p+1}+L^{(p)}
$$

thus according to the extension result 1.H.A.2, we have to exhibit a metric on the bundle

$$
F:=L_{p+1}+L^{(p)}+k L^{(q)}+q A
$$

for which the curvature conditions are satisfied, and such that the family of sections above are $L^{2}$ with respect to it. We define

$$
\varphi_{\delta, \varepsilon}^{(k q+p+1)}:=(1-\delta) \varphi_{L_{p+1}}+\delta \widetilde{\varphi}_{L_{p+1}}+(1-\varepsilon) \varphi^{(k q+p)}+\varepsilon q\left(k \varphi_{T}+\varphi_{A}+\frac{1}{q} \widetilde{\varphi}_{L^{(p)}}\right)
$$

and we check now the conditions that the parameters $\varepsilon, \delta$ have to satisfy.

We have to absorb the negativity in the smooth curvature terms in (46), and the one from $T$. The Hessian of the term

$$
1 / 3 \varphi_{A}+\frac{1}{q} \widetilde{\varphi}_{L^{(p)}}
$$

is assumed to be positive by $\left(\mathbf{U}_{4}\right), \dagger^{3}$, but we also have a negative contribution

$$
-k C_{T} \Theta_{A}
$$

induced by the current $T$. However we remark that we have

$$
k C_{T}<1 / 3
$$

by the hypothesis of the claim, and then the curvature of the metric defined in (44) will be positive, provided that

$$
\delta<\varepsilon q
$$

again by $\left(\dagger^{3}\right)$.

Let us check next the $L^{2}$ condition ; we have to show that the integral below in convergent

$$
\int_{\bar{S}} \frac{\left|s^{\otimes k} \otimes s_{j}^{(p+1)}\right|^{2}}{\left(\sum_{r}\left|s^{\otimes k} \otimes s_{r}^{(p)}\right|^{2}\right)^{1-\varepsilon}} \exp \left(-(1-\delta) \varphi_{L_{p+1}}-k q \varepsilon \varphi_{T}\right) d V .
$$

This is equivalent with

$$
\int_{\bar{S}}|s|^{2 \varepsilon k} \exp \left(-(1-\delta) \varphi_{L_{p+1}}-k q \varepsilon \varphi_{T}\right) d V<\infty .
$$

In order to show the above inequality, we use the same trick as before : the vanishing set of the section $s$ as in $\left(\mathbf{U}_{\mathbf{3}}\right)$ will allow us to apply the integrability lemma-the computations are strictly identical with those discussed in the point 3) above, but we give here some details. 
By the vanishing properties of the section $s$, the finiteness of the previous integral will be implied by the inequality

$$
\int_{\bar{S}} \exp \left(-(1-\delta) \varphi_{L_{p+1}}-k \varepsilon q\left(\varphi_{T}-\sum_{j \in J \backslash I} \rho^{j} \varphi_{Y_{j}}-\sum_{j \in I} \alpha^{j} \varphi_{Y_{j}}\right)\right) d V<\infty .
$$

In the first place, we have to keep the poles of $k \varepsilon q T$ "small" in the expression of the metric (46), thus we impose

$$
k \varepsilon q \leq \varepsilon_{0}
$$

The hypothesis in the integrability lemma also require

$$
1-\delta+\varepsilon k q C_{T}^{\prime}<1
$$

because of the contribution of the common part of Supp $L_{p+1}$ and $T$. Combined with the previous relations, the conditions for the parameters become

$$
\varepsilon k q C_{T}^{\prime}<\delta<\varepsilon q<\varepsilon_{0} / k .
$$

In conclusion, we can choose the parameters $\varepsilon, \delta$ so that the integrability/positivity conditions in the extension theorem are verified ; for example, we can take

- $\varepsilon:=\frac{\varepsilon_{0}}{2 k q}$

and

- $\delta:=\left(1+k C_{T}^{\prime}\right) \frac{\varepsilon_{0}}{4 k}$.

Finally, let us indicate how to perform the induction step if $p=q-1$ : we consider the family of sections

$$
s^{k+1} \otimes s_{j}^{(0)} \in H^{0}\left(\bar{S},(k+1) L^{(q)}+q A_{\mid \bar{S}}\right),
$$

In the case under consideration, we have to exhibit a metric on the bundle

$$
L_{q}+L^{(q-1)}+k L^{(q)}+q A
$$

however, this is easier than before, since we can simply take

$$
\varphi^{q(k+1)}:=q \varphi_{\bar{\Delta}_{2}}+\varphi^{(k q+q-1)}
$$

where the metric on $\bar{\Delta}_{2}$ is induced by the decomposition in $\left(\mathbf{U}_{\mathbf{1}}\right)$. With this choice, the curvature conditions are satisfied ; as for the $L^{2}$ ones, we remark that we have

$$
\begin{aligned}
& \int_{\bar{S}} \frac{\left|s^{k+1} \otimes s_{j}^{(0)}\right|^{2}}{\left(\sum_{r}\left|s^{k} \otimes s_{r}^{(q-1)}\right|^{2}\right)} \exp \left(-q \varphi_{\Delta_{2}}\right) d V< \\
&<C \int_{\bar{S}} \frac{\left|s \otimes s_{j}^{(0)}\right|^{2}}{\left(\sum_{r}\left|s_{r}^{(q-1)}\right|^{2}\right)} \exp \left(-q \varphi_{\Delta_{2}}\right) d V \leq C \int_{\bar{S}} \exp \left(-q \varphi \widehat{\Lambda}_{2}\right) d V<\infty
\end{aligned}
$$

where the last relation holds because the vanishing properties of $S$ and of the fact that $q \nu<1$ (cf. $\left(\mathbf{U}_{\mathbf{3}}\right)$. The proof of the extension claim is therefore finished. 


\section{$\S 1 . H . B$ Uniformity properties of $\left(K_{\widehat{X}}+S+\widehat{L}_{\eta}\right)_{\eta>0}$}

We come back now to the family of approximations $\left(\widehat{L}_{\eta}\right)$ we have produced in the paragraph $1 . \mathrm{F}$; our main concern in this paragraph will be to derive its uniformity properties, in order to apply the previous considerations. We list them below ; the constant $C$ which appear in the next statement is independent of $\eta$.

$\left(\mathbf{P}_{1}\right)$ There exist a closed $(1,1)$-current $\Theta_{\eta} \in\left\{K_{\widehat{X}}+S+\widehat{L}_{\eta}\right\}$ such that :

$\left(\mathbf{P}_{1.1}\right)$ It has logarithmic poles ;

$\left(\mathbf{P}_{\mathbf{1 . 2}}\right)$ It is greater than $-C \frac{\eta}{q_{\eta}} \omega ;$

$\left(\mathbf{P}_{1.3}\right)$ Its restriction to $S$ is well defined, and we have

$$
\Theta_{\eta \mid S}=\sum_{j \in J} \theta_{\eta}^{j}\left[Y_{j \mid S}\right]+R_{\eta, S}
$$

Moreover, the generic Lelong number of $R_{\eta, S}$ along $Y_{j \mid S}$ is zero, for any $j \in J$ and $\theta_{\eta}^{j} \leq \rho_{\eta}^{\infty, j}+C \frac{\eta}{q_{\eta}}$

$\left(\mathbf{P}_{\mathbf{2}}\right)$ The bundle $\widehat{L}_{\eta}$ can be endowed with a metric whose curvature current is given by

$$
\sum_{j \in J} \nu_{\eta}^{j}\left[Y_{j}\right]+\widehat{\Lambda}_{L}+\Xi(\eta)
$$

where $\Xi(\eta)$ is non-singular and greater than $-C \eta / q_{\eta}$; we equally have $Y_{j} \cap Y_{i}=\emptyset$, if $i \neq j$. As for $\widehat{\Lambda}_{L}$, we have the next important property : there exist a smooth, projective manifold $\widetilde{X}$ and a birational map $\mu_{1}: \widehat{X} \rightarrow \widetilde{X}$ such that

$$
\widehat{\Lambda}_{L} \geq \mu_{1}^{\star} \widetilde{\omega}
$$

where $\widetilde{\omega}$ is a Kähler metric on $\widetilde{X}$ and $S$ is not $\mu_{1}$-exceptional (see the proposition 1.D.1.).

$\left(\mathbf{P}_{\mathbf{3}}\right)$ The section $s_{\eta} \in H^{0}\left(S, q_{\eta}\left(K_{S}+\widehat{L}_{\eta}\right)\right)$ vanishes along the divisor

$$
q_{\eta}\left(\sum_{j \in J \backslash I} \rho_{\eta}^{\infty, j}\left[Y_{j \mid S}\right]+\sum_{j \in I} \nu_{\eta}^{j}\left[Y_{j \mid S}\right]\right)
$$

for all $0<\eta \ll 1$

The property $\left(\mathbf{P}_{\mathbf{3}}\right)$ is a simple recapitulation of facts which were completely proved during the previous paragraphs; let us give few details concerning $\left(\mathbf{P}_{\mathbf{1}}\right)$ and $\left(\mathbf{P}_{\mathbf{2}}\right)$.

By using the regularization theorem of Demailly, we have obtained in the section 1.E a family of currents with $\log$ poles $\Theta_{\varepsilon} \in\left\{K_{\widehat{X}}+S+\widehat{L}\right\}$ such that $\Theta_{\varepsilon} \geq-\varepsilon \omega$, and whose restriction to $S$ equals

$$
\Theta_{\varepsilon \mid S}=\sum_{j \in J} \rho^{\varepsilon, j} Y_{j \mid S}+R_{\varepsilon}
$$


We first "move" the current $\Theta_{\varepsilon}$ in the class $\left\{K_{\widehat{X}}+S+\widehat{L}_{\eta}\right\}$ by a smooth form ; we denote by $\Theta_{\varepsilon, \eta}$ the result, and we remark that we have

$$
\Theta_{\varepsilon, \eta} \geq-\left(\varepsilon+C \frac{\eta}{q_{\eta}}\right) \omega
$$

by the approximation lemma 1.F.1. Next, we take $\varepsilon$ small enough such that :

- $\varepsilon \leq C \frac{\eta}{q_{\eta}}$;

- $\left|\rho_{\eta}^{\infty, j}-\rho^{\varepsilon, j}\right| \leq C \frac{\eta}{q_{\eta}}$ for each $j \in J$.

With this choice, the corresponding current will be our $\Theta_{\eta}$; it satisfy all the requirements in the $\left(\mathbf{P}_{\mathbf{1}}\right)$ above.

The curvature current in the first Chern class of $\widehat{L}_{\eta}$ can be obtained as in the section 1.G ; nevertheless, we will give here the full details of the construction. We consider the decomposition

$$
\widehat{L}_{\eta}=L+\left(\widehat{L}_{\eta}-L\right)
$$

and we remark that by the approximation lemma 1.F.1, the second term of the previous decomposition can be endowed with a non-singular metric whose curvature form is greater than $-C \eta / q_{\eta}$. We replace the coefficients $\nu^{j}$ of $L$ (see the property 1.D.1) with their rational approximations $\nu_{\eta}^{j}$ (cf. 1.F.1), and the negativity of the error term is again dominated by $-C \eta / q_{\eta}$; thus, the claim $\left(\mathbf{P}_{\mathbf{2}}\right)$ is verified.

We will establish now the relations between $\left(\mathbf{U}_{\mathbf{0}}\right)-\left(\mathbf{U}_{\mathbf{4}}\right)$ and $\left(\mathbf{P}_{\mathbf{1}}\right)-\left(\mathbf{P}_{\mathbf{3}}\right)$.

- $\bar{X}:=\widehat{X}, \bar{S}=S$ and $\bar{L}:=\widehat{L}_{\eta}$; the corresponding coefficients/forms are $\alpha^{j}:=\nu_{\eta}^{j}$ and $\left\{\bar{\Lambda}_{2}\right\}:=\left\{\widehat{\Lambda}_{L}+\Xi(\eta)\right\}$ (we will choose later the representative corresponding to $\bar{\Lambda}_{2}$ ).

- The hypersurfaces $\left(Y_{j}\right)_{j \in J}$ are the same for all $\eta>0$.

- $T:=\Theta_{\eta}$, therefore $\bar{\theta}^{j}:=\theta_{\eta}^{j}$.

- $q:=q_{\eta}$ and $s:=s_{\eta}$; thus we have $C_{T}^{\prime}:=C \frac{\eta}{q_{\eta}}$, where we insist on the fact that $C$ does not depend on $\eta$.

In order to apply the claim we still have to clarify the following points : we have to identify the decomposition of $\widehat{L}_{\eta}$ as in $\left(\mathbf{U}_{\mathbf{1}}\right)$ and "trade" $\widehat{\Lambda}_{L}+\Xi(\eta)$ for a Kähler current, with an estimate for $\nu$ as it is required by $\left(\mathbf{U}_{\mathbf{1}}\right)$ and $\left(\mathbf{U}_{\mathbf{3}}\right)$. Also, we have to choose the bundle $A$ with the property $\left(\mathbf{U}_{4}\right)$. This will be discussed along the next lines.

We first remark that we have the next decomposition

$$
\widehat{L}_{\eta} \equiv \sum_{j \in J \backslash I} \nu_{\eta}^{j}\left[Y_{j}\right]+\sum_{j \in I} \nu_{\eta}^{j}\left[Y_{j}\right]+\widehat{\Lambda}_{L}+\Xi(\eta) ;
$$

the coefficients corresponding to the indexes $j \in J$ are positive, as soon as $\eta$ is small enough.

Let us introduce the next notations : 
- $\Delta_{1}:=\sum_{j \in J \backslash I} \nu_{\eta}^{j}\left[Y_{j}\right]$. It is an effective $\mathbb{Q}$-bundle whose critical exponent is greater than 1 , and such that the multiple $q_{\eta} \nu_{\eta}^{j}$ is a positive integer strictly smaller than $q_{\eta}$, for each $j \in J \backslash I$;

- $\Delta_{2}:=\sum_{j \in I} \nu_{\eta}^{j}\left[Y_{j}\right]+\widehat{\Lambda}_{L}+\Xi(\eta)$. It is equally a $\mathbb{Q}$-bundle whose critical exponent is greater than 1 and such that $q_{\eta} \Delta_{2}$ is integral. One of the facts which will be relevant in what follows is that the section $s_{\eta}$ vanishes along the singular part of $q_{\eta} \Delta_{2}$.

By the property $\left(\mathbf{P}_{\mathbf{2}}\right)$, we can find a representative of the class $\left\{\widehat{\Lambda}_{L}\right\}$ which dominates a Kähler metric (see also the previous section) ; in general we cannot avoid that this representative acquire some singularities. However, in the present context we will show that there exist a Kähler current in the above class which is "restrictable" to $S$. It is at this point that we use the full force of the property $\left(\mathbf{P}_{\mathbf{2}}\right)$.

Indeed, we consider the exceptional divisors $\left(E_{j}\right)$ of the map $\mu_{1}$ (see the paragraph 1.D) ; the hypersurface $S$ do not belong to this set, and then the class

$$
\widehat{\Lambda}_{L}-\sum_{j} \varepsilon^{j} E_{j}
$$

is ample on $\widehat{X}$, for some positive reals $\varepsilon^{j}$. Once a set of such parameters is chosen, we fix a Kähler form

$$
\Omega \in\left\{\widehat{\Lambda}_{L}-\sum_{j} \varepsilon^{j} E_{j}\right\}
$$

and for each $\delta \in[0,1]$ we define

$$
\widehat{\Lambda}_{L, \delta}:=(1-\delta) \widehat{\Lambda}_{L}+\delta\left(\Omega+\sum_{j} \varepsilon^{j} E_{j}\right) \in\left\{\widehat{\Lambda}_{L}\right\} .
$$

For each $\eta>0$, there exist $\delta>0$ such that

$M_{3}$ ) The current $\widehat{\Lambda}_{L, \delta}+\Xi(\eta)$ dominates a Kähler form on $\widehat{X}$;

$M_{4}$ ) The Lelong number of the restriction $\widehat{\Lambda}_{L, \delta \mid S}$ at each point of $S$ does not exceed $C \frac{\eta}{q_{\eta}}$, where $C$ is a constant independent of $\eta$.

One can take $\delta:=\varepsilon_{0}^{-1} \frac{\eta}{q_{\eta}}$ where $\varepsilon_{0}$ is small (but fixed) and the properties $M_{3}$ ) and $\left.M_{4}\right)$ are clearly satisfied, since the negative part of $\Xi(\eta)$ is given by the property $\left(\mathbf{P}_{\mathbf{2}}\right)$ above. We denote by $\widehat{\Lambda}_{L, \eta}$ the corresponding current, and we observe that it satisfy the next property as well.

$M_{5}$ ) The restriction of $\widehat{\Lambda}_{L, \eta}$ to $S$ is well defined.

Thus, the $\mathbb{Q}$-divisor $\Delta_{2}$ is linearly equivalent to

$$
\Delta_{2} \equiv \sum_{j \in I} \nu_{\eta}^{j}\left[Y_{j}\right]+\widehat{\Lambda}_{L, \eta}+\Xi(\eta)
$$


it is a Kähler current and we have $q_{\eta} \max \nu\left(\widehat{\Lambda}_{L, \eta \mid S}, s\right) \ll 1$, for $\eta$ small enough ; therefore, the first inequality in $\left(\mathbf{U}_{\mathbf{3}}\right)$ is satisfied.

Precisely as in [4], [15], [18] there exist a decomposition

$$
q_{\eta} \Delta_{1}=L_{1}+\ldots+L_{q_{\eta}-1}
$$

such that for each $m=1, \ldots, q_{\eta}-1$, we have

$$
L_{m}:=\sum_{j \in I_{m} \subset J \backslash I} Y_{j}
$$

We denote by $L_{q_{\eta}}:=q_{\eta} \Delta_{2}$ and

$$
L^{(p)}:=p\left(K_{X}+S\right)+L_{1}+\ldots+L_{p}
$$

where $p=1, \ldots, q_{\eta}$. By convention, $L^{(0)}$ is the trivial bundle.

Finally, it is possible to find an ample bundle $\left(A, h_{A}\right)$ independent of $\eta$ whose curvature form is positive enough such that the next relations hold.

(†) For each $0 \leq p \leq q_{\eta}-1$, the bundle $L^{(p)}+q_{\eta} A$ is generated by its global sections, which we denote by $\left(s_{j}^{(p)}\right)$.

$\left(\dagger^{2}\right)$ Any section of the bundle $L^{\left(q_{\eta}\right)}+q_{\eta} A_{\mid S}$ admits an extension to $\tilde{X}$.

$\left(\dagger^{3}\right)$ We endow the bundle corresponding to $\left(Y_{j}\right)_{j \in J}$ with a non-singular metric, and we denote by $\widetilde{\varphi}_{m}$ the induced metric on $L_{m}$. Then for each $m=1, \ldots, q_{\eta}$, the functions

$$
\widetilde{\varphi}_{L_{m}}+1 / 3 \varphi_{A}
$$

are strictly psh.

$\left(\dagger^{4}\right)$ For any $\eta>0$ we have

$$
\Theta_{\eta} \geq-\frac{\eta}{q_{\eta}} \Theta_{A} .
$$

thus $C_{T}:=\frac{\eta}{q_{\eta}}$.

In other words, the bundle " $A$ " in $\mathbf{U}_{4}$ will be $A$ in the present context.

Remark. Concerning the construction and the properties of $\widehat{\Lambda}_{L, \delta}$, we recall the very nice result in [17], stating that if $D$ is an $\mathbb{R}$-divisor which is nef and big, then its associated augmented base locus can be determined numerically. 


\section{§1.H.C End of the proof}

We show next that the sections $s_{\eta}$ can be lifted to $\widehat{X}$ as soon as $\eta$ is small enough, by using the claim proved in 1.H.A.

Indeed, we consider the extensions $U_{j}^{\left(k q_{\eta}\right)}$ of the sections $s_{\eta}^{\otimes k} \otimes s_{j}^{(0)}$; they can be used to define a metric on the bundle

$$
k q_{\eta}\left(K_{\widehat{X}}+S+\widehat{L}_{\eta}\right)+q_{\eta} A
$$

whose $k q_{\eta}^{\text {th }}$ root it is defined to be $h_{k}^{(\eta)}$.

As usual, we write the bundle we are interested in i.e. $q_{\eta}\left(K_{\widehat{X}}+S+\widehat{L}_{\eta}\right)$ as an adjoint bundle ; we have

$$
\begin{aligned}
q_{\eta}\left(K_{\widehat{X}}+S+\widehat{L}_{\eta}\right) & =K_{\widehat{X}}+S+\left(q_{\eta}-1\right)\left(K_{\widehat{X}}+S+\widehat{L}_{\eta}\right)+\widehat{L}_{\eta}= \\
& =K_{\widehat{X}}+S+\left(q_{\eta}-1\right)\left(K_{\widehat{X}}+S+\widehat{L}_{\eta}+1 / k A\right)+\widehat{L}_{\eta}-\frac{q_{\eta}-1}{k} A
\end{aligned}
$$

Given the extension theorem 1.H.A.2, we need to construct a metric on the bundle

$$
\left(q_{\eta}-1\right)\left(K_{\widehat{X}}+S+\widehat{L}_{\eta}+1 / k A\right)+\widehat{L}_{\eta}-\frac{q_{\eta}-1}{k} A .
$$

On the first factor of the above expression we will use $\left(q_{\eta}-1\right) \varphi_{k}^{(\eta)}$ (that is to say, the $\left(q_{\eta}-1\right)^{\text {th }}$ power of the metric given by $\left.h_{k}^{(\eta)}\right)$.

We endow the bundle $\widehat{L}_{\eta}$ with a metric whose curvature is given by the expression

$$
\sum_{j \in J \backslash I} \nu_{\eta}^{j}\left[Y_{j}\right]+\sum_{j \in I} \nu_{\eta}^{j}\left[Y_{j}\right]+\widehat{\Lambda}_{L, \delta}+\Xi(\eta) ;
$$

here we take $\delta$ independent of $\eta$, but small enough such that the critical exponent of the resulting metric on $\widehat{L}_{\eta \mid S}$ is still greater than 1 . Finally, we multiply with the $\frac{q_{\eta}-1}{k}$ times $h_{A}^{-1}$.

The corresponding constants $C_{T}$, respectively $C_{T}^{\prime}$ in 1.H.A are in the present context $\frac{\eta}{q_{\eta}}$, respectively $C \frac{\eta}{q_{\eta}}$; thus by the claim, we are free to choose $k$ e.g. such that $k=q_{\eta}\left[\eta^{-1 / 2}\right]$ (where $[x]$ denotes the integer part of the real $x$ ). Then the metric above is not identically $\infty$ when restricted to $S$, and its curvature will be strongly positive as soon as $\eta \ll 1$. Indeed, the curvature of $\widehat{L}_{\eta}$ is greater than a Kähler metric on $\widehat{X}$ which is independent of $\eta$ because of the factor $\widehat{\Lambda}_{L, \delta}$.

Moreover, the $L^{2}$ conditions in the theorem 1.H.A.2 are satisfied, since the norm of the section $s_{\eta}$ with respect to the metric $q_{\eta} \varphi_{k}^{(\eta)}$ is pointwise bounded, and since the critical exponent of the metric on $\widehat{L}_{\eta \mid S}$ is greater than 1 .

In conclusion, we obtain an extension of the section $s_{\eta}$, and the theorem 0.1 is completely proved. 
Remark. The exact vanishing properties of $s_{\eta}$ given by the proposition 1.G.1 are crucial. Indeed, assume that instead of the divisor

$$
q_{\eta}\left(\sum_{j \in J \backslash I} \rho_{\eta}^{\infty, j}\left[Y_{j \mid S}\right]+\sum_{j \in I} \nu_{\eta}^{j}\left[Y_{j \mid S}\right]\right)
$$

the section $s_{\eta}$ only vanishes along

$$
\left(q_{\eta}-1\right)\left(\sum_{j \in J \backslash I} \rho_{\eta}^{\infty, j}\left[Y_{j \mid S}\right]+\sum_{j \in I} \nu_{\eta}^{j}\left[Y_{j \mid S}\right]\right) .
$$

This may look innocent, since $q_{\eta} \rightarrow \infty$ anyway, but we remark that under these circumstances we have

$$
C_{T}^{\prime}=C \frac{\eta}{q_{\eta}}+\max _{j}\left\{\frac{\rho^{\infty, j}}{q_{\eta}}\right\}
$$

and the whole extension process collapse, since we cannot insure

$$
\frac{q_{\eta}}{k} \rightarrow 0
$$

anymore.

\section{$\S 2$. Metrics with minimal singularities and holomorphic sec- tions}

In this section we will consider the following geometric context. Let $X$ be a nonsingular, projective $n$-dimensional manifold, and let $L \rightarrow X$ be a $\mathbb{Q}$-line bundle with the following metric property :

$(\bullet)$ The $(1,1)$-class $c_{1}(L)$ contains a Kähler current $\Theta_{L}$ whose critical exponent is strictly greater than 1 .

We consider the following graded algebra

$$
\mathcal{R}(X, L):=\bigoplus_{k \in q \mathbb{Z}_{+}} H^{0}\left(X, k\left(K_{X}+L\right)\right)
$$

where $q \in \mathbb{Z}_{+}$is a positive integer such that $q L$ is a line bundle. In order to study its properties, we assume that a non-singular metric $\widetilde{h}$ on $K_{X}+L$ is given, and for any

$$
u \in H^{0}\left(X, k\left(K_{X}+L\right)\right)
$$

we will denote by $|u|_{k}$ the norm of $u$ with respect to the metric $\widetilde{h}^{\otimes k}$.

In the paragraph 2.A below we define a metric with minimal singularities which is adapted to the ring $\mathcal{R}(X, L)$. For some technical reasons (which will only appear in the second paragraph 2.B), we are forced to take into account the sections of the multiples 
of $K_{X}+L$ twisted with a topologically trivial line bundle, even if our ultimate goal would be to understand the structure of the ring $\mathcal{R}(X, L)$.

Next, we will consider the relative threshold of the minimal metric with respect to a metric given by a finite number of sections of the multiples of $K_{X}+L$; if the singularities of these metrics do not coincide, then the non-vanishing theorem will provide us with an $\mathbb{R}$-section of $K_{X}+L$ which has precisely the same vanishing order along some divisor, say $S$, of a modification of $X$ as the metric with minimal singularities (compare with [39]). The results we obtain in the paragraph 2.A will show in particular that the said vanishing order is a rational number.

\section{§2.A Metrics with minimal singularities}

Along the following lines, we will only consider the case of adjoint bundles, since it is in this setting that the main properties we will establish afterwards hold ; however, one could define the objects below in a more general context. A general reference for the notions discussed in this subsection is the article [14].

Let $\rho \rightarrow X$ be a topologically trivial line bundle, endowed with a metric $h_{\rho}$ whose curvature form is equal to zero. Given

$$
u \in H^{0}\left(X, k\left(K_{X}+L\right)+\rho\right)
$$

we denote by $|u|_{k, \rho}^{2}$ the poinwise norm of $u$, measured with the $\widetilde{h}^{k}$ twisted with $h_{\rho}$. We introduce the following class of functions on $X$

$$
\mathcal{F}:=\left\{f=\frac{1}{k} \log |u|_{k, \rho}^{2}: k \in q \mathbb{Z}_{+}, u \in H^{0}\left(X, k\left(K_{X}+L\right)+\rho\right) \text {, s.t. } \sup _{X}|u|_{k, \rho}^{2}=1\right\}
$$

and we remark that we have

$$
\Theta_{\widetilde{h}}\left(K_{X}+L\right)+\frac{\sqrt{-1}}{2 \pi} \partial \bar{\partial} f \geq 0
$$

for any $f \in \mathcal{F}$ (since the curvature of $\rho$ with respect to $h_{\rho}$ is equal to zero). Thus the curvature current associated to the metric

$$
\exp (-f) \widetilde{h}
$$

on the bundle $K_{X}+L$ is positive.

We will denote by $h_{\text {min }}$ the metric on $K_{X}+L$ given by the smallest upper semicontinuous majorant of the family $\mathcal{F}$ above (see [14]). We denote by

$$
\Theta_{\min }:=\Theta_{h_{\min }}\left(K_{X}+L\right)
$$

the curvature current associated to this metric ; by definition we have

$$
|v|^{2} \exp \left(-k \varphi_{\min }\right) \leq O(1)
$$

for any $v \in H^{0}\left(X, k\left(K_{X}+L\right)+\rho\right)$. 
Next we fix a topologically trivial line bundle $\rho_{0}$ on $X$ and an integer $m_{0} \in q \mathbb{Z}_{+}$; we will construct another metric on the bundle $K_{X}+L$ as follows. We consider the set of potentials

$$
\mathcal{F}_{0}:=\left\{f=\frac{1}{k m_{0}} \log |u|_{k m_{0}, k \rho_{0}}^{2}: u \in H^{0}\left(X, k m_{0}\left(K_{X}+L\right)+k \rho_{0}\right), \sup _{X}|u|_{k m_{0}, k \rho_{0}}^{2}=1\right\}
$$

and we denote by $h_{\text {min }}^{\rho_{0}}$ the metric on $K_{X}+L$ given by the smallest upper semicontinuous majorant of the family $\mathcal{F}^{\rho_{0}}$; let $\Theta_{\text {min }}^{\rho_{0}}$ be the associated curvature current.

We state now the main result of this subsection.

2.A.1 Theorem. We have

$$
h_{\min }=h_{\min }^{\rho_{0}} .
$$

2.A.2 Remark. If the adjoint bundle $K_{X}+L$ is big, then the above result is a consequence of the regularization theorem [11]. Also, the results of Campana-Peternell (see [8]) suggest that the strict positivity of $L$ in the theorem above may be superfluous.

Proof (of the theorem 2.A.1). The relation

$$
\varphi_{\min } \geq \varphi_{\min }^{\rho_{0}}
$$

is implied by the definition ; in order to obtain an inequality in the opposite sense, we consider a section

$$
u_{1} \in H^{0}\left(X, m_{1}\left(K_{X}+L\right)+\rho_{1}\right)
$$

whose norm is smaller than 1.

As a consequence of an argument due to Shokurov (already employed in the section 1.G), we have the next statement.

2.A.2 Lemma. For any $k \in \mathbb{Z}_{+}$, there exist a section

$$
u_{k} \in H^{0}\left(X, k m_{1} m_{0}\left(K_{X}+L\right)+k m_{1} \rho_{0}\right)
$$

such that the next integral condition is satisfied

$$
\int_{X} \frac{c_{k} u_{k} \wedge \overline{u_{k}} \exp \left(-k m_{1} \varphi_{\rho_{0}}\right)}{\left(c_{1} u_{1} \wedge \overline{u_{1}}\right)^{\frac{k m_{1} m_{0}-1}{m_{1}}} \exp \left(-\frac{k m_{1} m_{0}-1}{m_{1}} \varphi_{\rho_{1}}\right)} \exp \left(-\varphi_{L}\right) d \lambda=1
$$

In the lemma above we denote by $\varphi_{L}$ the local weight of any metric on $L$ which satisfy the properties at the beginning of 2.A : the corresponding curvature is a Kähler current whose critical exponent is greater than 1 . The quantities $c_{k}$ and $c_{1}$ are the usual ones, such that the wedge at the denominator and numerator in the previous lemma are 
reals. We remark that the quantity under the integral sign in the above formula is a globally defined measure.

Proof. For any positive integer $k$, we consider the bundles

$$
k m_{1} m_{0}\left(K_{X}+L\right)+k m_{1} \rho_{0}
$$

and

$$
k m_{1} m_{0}\left(K_{X}+L\right)+k m_{0} \rho_{1}
$$

as well as the multiplier ideal

$$
I_{k}:=\mathcal{J}\left(\left(k m_{1} m_{0}-1\right) \log \left|u_{1}\right|_{m_{1}, \rho_{1}}^{\frac{2}{m_{1}}}+\varphi_{L}\right)
$$

We have

$$
\chi\left(\left(k m_{1} m_{0}\left(K_{X}+L\right)+k m_{1} \rho_{0}\right) \otimes I_{k}\right)=\chi\left(X,\left(k m_{1} m_{0}\left(K_{X}+L\right)+k m_{0} \rho_{1}\right) \otimes I_{k}\right)
$$

by the usual arguments (i.e. the existence of a finite and free resolution of the multiplier sheaf $I_{k}$, and the fact that $\rho_{j}$ are topologically trivial).

Thanks to the Kawamata-Viehweg-Nadel vanishing theorem we equally know that the respective higher cohomology groups are equal to zero, so in conclusion

$H^{0}\left(X,\left(k m_{1} m_{0}\left(K_{X}+L\right)+k m_{1} \rho_{0}\right) \otimes I_{k}\right)=H^{0}\left(X,\left(k m_{1} m_{0}\left(K_{X}+L\right)+k m_{0} \rho_{1}\right) \otimes I_{k}\right)$.

We claim now that the section $u_{1}^{\otimes k m_{0}}$ belong to the right hand side cohomology group in (54). To verify this claim, we have to show that the following integral converge

$$
\int_{X}\left|u_{1}\right|^{\frac{2}{m_{1}}} \exp \left(-\varphi_{L}\right) d \lambda
$$

and indeed this is the case, since the critical exponent of the current $\Theta_{L}$ is greater than 1.

Therefore we infer the existence of a non-identically zero section

$$
u_{k} \in H^{0}\left(X,\left(k m_{1} m_{0}\left(K_{X}+L\right)+k m_{1} \rho_{0}\right) \otimes I_{k}\right)
$$

By the construction of the ideal $I_{k}$, we see that we can normalize the section $u_{k}$ such that the integral condition (54) is satisfied; the lemma 2.A.2 is therefore proved.

The finiteness of (54) show the existence of a section

$$
v_{k} \in H^{0}\left(X, m_{1}\left(K_{X}+L\right)+k m_{1} \rho_{0}-k m_{0} \rho_{1}\right)
$$

such that

$$
u_{k}=u_{1}^{\otimes\left(k m_{0}-1\right)} \otimes v_{k}
$$


the integral relation (54) become

$$
\int_{X} \frac{c_{1} v_{k} \wedge \bar{v}_{k}}{\left(c_{1} u_{1} \wedge \bar{u}_{1}\right)^{\frac{m_{1}-1}{m_{1}}}} \exp \left(-\varphi_{L}-k m_{1} \varphi_{\rho_{0}}+\left(k m_{0}-1+1 / m_{0}\right) \varphi_{\rho_{1}}\right)=1
$$

We will use the family of sections $\left(u_{k}\right)_{k \in \mathbb{Z}_{+}}$in order to compare $\varphi_{\min }$ and $\varphi_{\min }^{\rho_{0}}$. A specific normalization was chosen for the sections defining the potentials in $\mathcal{F}$; thus, we have to estimate the sup norm of $u_{k}$ along the next lines. Our main technical tools will be the standard convexity properties of the psh functions ; we use the same notation " $C$ " for all the constants which will occur during the following computations, even if they are not the same inside the same line, as long as they do not depend on $k$.

We define

$$
\exp \left(f_{k}\right):=\left|v_{k}\right|_{m_{1}, k m_{1} \varphi_{\rho_{0}}-\left(k m_{0}-1\right) \varphi_{\rho_{1}}}^{2}
$$

the next step in our proof is to show the existence of a positive constant $C=C\left(m_{1}\right)$ large enough, so that we have

$$
\sqrt{-1} \partial \bar{\partial} f_{k} \geq-C \omega
$$

and moreover

$$
-\log C \leq \max _{X} f_{k} \leq \log C
$$

The inequality (64) is a consequence of the fact that the curvature form of the metrics on the bundles $\rho_{j}$ is equal to zero ; let us give some explanations about (65). By using the notations introduced in (63), the equality (62) become

$$
\int_{X} \frac{\exp \left(f_{k}-f_{L}\right)}{\left|u_{1}\right|_{m_{1}, \rho_{1}}^{2 \frac{m_{1}-1}{m_{1}}}} d V_{\omega}=1
$$

where $f_{L}$ is the (global) distortion function between the metric $\varphi_{L}$ and the non-singular metric on $L$ induced by $\widetilde{h}$ on $K_{X}+L$ and $\operatorname{det}(\omega)$ on $-K_{X}$. The section $u_{1}$ is normalized such that

$$
\max _{X}\left(\left|u_{1}\right|_{m_{1}, \rho_{1}}\right)=1
$$

and then the right hand side part of (65) is a consequence of the mean inequality for the psh functions.

In order to obtain the first inequality, we consider a log-resolution $\mu: \widehat{X} \rightarrow X$ of the function

$$
\psi:=f_{L}+\frac{m_{1}-1}{m_{1}} \log \left|u_{1}\right|_{m_{1}, \rho_{1}}^{2}
$$

and we have

$$
\psi \circ \mu:=\widehat{\psi}+\sum_{j \in J} a_{\psi}^{j} \log \left|s_{j}\right|^{2}
$$


as well as

$$
K_{\widehat{X} / X}:=\sum_{j \in J} a_{\widehat{X} / X}^{j}\left[W_{j}\right]
$$

where $\widehat{\psi}$ is a smooth function globally defined on $\widehat{X}$, and the hypersurfaces

$$
W_{j}:=\left(s_{j}=0\right)
$$

have normal crossings. We stress at this point on the fact that $\mu$ does not depend on $k$.

For each positive integer $k$ we decompose the inverse image of $f_{k}$ as follows

$$
f_{k} \circ \psi:=\widehat{f}_{k}+\sum_{j \in J} a_{k}^{j} \log \left|s_{j}\right|^{2}
$$

where $\widehat{f}_{k}$ is non singular along any of $\left(W_{j}\right)$, and we observe that there exist a metric $\widehat{\omega}$ on $\widehat{X}$ such that we have

$$
\sqrt{-1} \partial \bar{\partial} \widehat{f}_{k} \geq-\widehat{\omega}
$$

for any $k$-this is a direct consequence of the relation (64). With this notations, the equality (66) become

$$
\int_{X} \exp \left(\widehat{f_{k}}-\widehat{\psi}+\sum_{j \in J}\left(a_{k}^{j}+a_{\widehat{X} / X}^{j}-a_{\psi}^{j}\right) \log \left|s_{j}\right|^{2}\right) d V_{\widehat{\omega}}=C
$$

for some constant $C>0$, uniform with respect to $k$ (which appear instead of 1 because of $(67))$. The finiteness of the above integral show that we have

$$
a_{k}^{j}+a_{\widehat{X} / X}^{j}-a_{\psi}^{j}>-1
$$

for any $j \in J$. We remark that the positive reals $a_{k}^{j}$ defined above are in fact integers, since they correspond to the vanishing order of the inverse image of $v_{k}$ along $W_{j}$. Therefore we obtain

$$
a_{k}^{j} \geq\left[a_{\psi}^{j}-a_{\widehat{X} / X}^{j}\right]
$$

for all $j \in J$ and we re-write the formula (68) as follows

$$
\int_{X} \exp \left(\tilde{f}_{k}-\widetilde{\psi}\right) d V_{\widehat{\omega}}=C
$$

where we use the following notations

$$
\widetilde{f}_{k}:=\widehat{f}_{k}+\sum_{j \in J}\left(a_{k}^{j}-\left[a_{\psi}^{j}-a_{\widehat{X} / X}^{j}\right]\right) \log \left|s_{j}\right|^{2}
$$

and

$$
\widetilde{\psi}:=\widehat{\psi}+\sum_{j \in J}\left\{a_{\psi}^{j}-a_{\widehat{X} / X}^{j}\right\} \log \left|s_{j}\right|^{2}
$$


We remark that the equality (69) is similar to (66), but in addition multiplier ideal of the function $\widetilde{\psi}$ is trivial.

Let

$$
\widetilde{C}_{k}:=\max _{\widehat{X}}\left(\widetilde{f}_{k}\right)
$$

as a consequence of the relations (67) and (69), there exist a positive constant $\widetilde{C}$ such that

$$
\widetilde{C}_{k} \leq C
$$

for any $k \in \mathbb{Z}_{+}$. The equality (69) imply

$$
C \exp \left(-\widetilde{C}_{k}\right)=\int_{X} \exp \left(\widetilde{f}_{k}-\widetilde{C}_{k}-\widetilde{\psi}\right) d V_{\widehat{\omega}}
$$

and thus

$$
\exp \left(-\widetilde{C}_{k}\right) \leq C \int_{X} \exp (-\widetilde{\psi}) d V_{\widehat{\omega}}:=C<\infty
$$

In conclusion, the sequence $\left(\widetilde{C}_{k}\right)$ is bounded from below as well.

By the usual properties of the quasi-psh functions (see e.g. [11], [12]), there exist a function $\widetilde{f}_{\infty} \in L^{1}(\widehat{X})$ such that

$$
\tilde{f}_{k} \rightarrow \tilde{f}_{\infty}
$$

as $k \rightarrow \infty$. This show in particular the validity of the inequality (65), since

$$
f_{k} \circ \mu=\widetilde{f}_{k}+\sum_{j \in J}\left[a_{\psi}^{j}-a_{\widehat{X} / X}^{j}\right] \log \left|s_{j}\right|^{2}
$$

and thus the sequence $\left(f_{k}\right)$ cannot tend to $-\infty$.

The important consequence of the previous considerations is the existence of a limit for the sequence $\left(f_{k}\right)$. The normalization of $u_{1}$ and the relation (56) show that

$$
\max _{X}\left|u_{k}\right|_{k m_{1} m_{0}, k m_{1} \rho_{0}} \leq e^{C\left(m_{1}\right)}
$$

and finally we get

$$
\begin{aligned}
\varphi_{\min }^{\rho_{0}} & \geq-\frac{C\left(m_{1}\right)}{k m_{1} m_{0}}+\frac{1}{k m_{1} m_{0}} \log \left|u_{k}\right|^{2} \geq \\
& \geq-\frac{C\left(m_{1}\right)}{k m_{1} m_{0}}+\frac{k m_{0}-1}{k m_{1} m_{0}} \log \left|u_{1}\right|^{2}+\frac{1}{k m_{1} m_{0}} f_{k} .
\end{aligned}
$$

We let $k \rightarrow \infty$; the first and the third term in the last inequality above tend to zero, and thus we get

$$
\varphi_{\min }^{\rho_{0}} \geq \frac{1}{m_{1}} \log \left|u_{1}\right|^{2}
$$

The section $u_{1}$ above is arbitrary, thus the theorem is proved. 


\section{§2.B Constructing sections with minimal vanishing order}

In this paragraph we would like to point out an important property of the zeroes of the $\mathbb{R}$-sections produced by 0.1 , in connection with Siu's proof of the finite generation problem (see [39], [40]). The same hypothesis/conventions as in the beginning of the section are in force ; in addition, given an integer $\alpha$ large enough, we consider the following truncation metric

$$
\varphi_{\alpha}:=\log \left(\sum_{k=1}^{\alpha} \varepsilon_{k} \sum_{j \in J_{k}}\left|f_{j}^{k}\right|^{\frac{2}{k}}\right)
$$

where $\varepsilon_{k}$ are positive real numbers, and $\left(f_{j}^{k}\right)$ are local expressions of a family of sections of $k\left(K_{X}+L\right)$; let $\Theta_{\alpha}$ be the corresponding current.

Precisely as in the paragraph 1 .C, we will consider $\mu: \widehat{X} \rightarrow X$ a log-resolution of the currents $\Theta_{L}$ and $\Theta_{\alpha}$; we have

$$
\mu^{\star}(\Delta)=\sum_{j \in J} a_{\Delta}^{j}\left[Y_{j}\right]
$$

and

$$
\mu^{\star}\left(\Theta_{L}\right)=\sum_{j \in J} a_{L}^{j}\left[Y_{j}\right]+\widehat{\Lambda}_{L}
$$

as well as

$$
\mu^{\star}\left(\Theta_{\alpha}\right)=\sum_{j \in J} a_{\alpha}^{j}\left[Y_{j}\right]+\widehat{\Lambda}_{\alpha}
$$

where $\widehat{\Lambda}_{L}$, respectively $\widehat{\Lambda}_{\alpha}$ are non-singular and semi-positive $(1,1)$-forms on $\widehat{X}$ which are positively defined at the generic point of this manifold. We consider next the inverse image of the minimal current via $\mu$ :

$$
\mu^{\star}\left(\Theta_{\min }\right)=\sum_{j \in J} a_{\min }^{j}\left[Y_{j}\right]+\widehat{\Lambda}_{\min }
$$

where $\widehat{\Lambda}_{\text {min }}$ is a closed positive current, whose generic Lelong numbers along the hypersurfaces $Y_{j}$ above is equal to zero. Moreover, by the definition of the minimal metric we have

$$
a_{\alpha}^{j} \geq a_{\min }^{j}
$$

for all $j \in J$. We equally have the pointwise inequality

$$
\varphi_{\widehat{\Lambda}_{\text {min }}} \geq \sum_{j \in J}\left(a_{\alpha}^{j}-a_{\text {min }}^{j}\right) \log \left|f_{j}\right|^{2}
$$


modulo an irrelevant constant.

The main result of the current subsection is the following.

2.B.1 Theorem. If at least one of the inequalities (75) is strict, then there exist a topologically trivial line bundle $\rho \rightarrow X$ and a section $u \in H^{0}\left(X, m\left(K_{X}+L\right)+\rho\right)$ such that the vanishing order of $\mu^{\star}(u)$ along $Y_{j_{0}}$ is precisely $m a_{\min }^{j_{0}}$ for some index $j_{0} \in J$. In particular, we have $a_{\min }^{j_{0}} \in \mathbb{Q}$.

We remark that if all the inequalities (75) are equalities, then (76) show that all the local potentials of the current $\widehat{\Lambda}_{\text {min }}$ are bounded. In other words, the metric with minimal singularities is equivalent with its truncation $\varphi_{\alpha}$, and this imply the finite generation of the ring associated to $K_{X}+L$, according to [39].

Proof. We consider the relative threshold associated to the following objects :

$$
\tau:=\sup \left\{t \in \mathbb{R}_{+}: \int_{\widehat{X}} \exp \left(t\left(\varphi_{\widehat{D}}-\varphi_{\alpha}\right)+\varphi_{\widehat{D}}+\varphi_{\widetilde{X} / X}-\varphi_{L} \circ \mu\right) d \lambda<\infty\right\}
$$

where we use the notation

$$
\widehat{D}:=\sum_{j \in J} a_{\min }^{j}\left[Y_{j}\right]
$$

We observe that $\tau$ verify the next relations

$$
0<\tau<\infty
$$

by the same arguments as in the proof of $0.1-$ we remark that the latter inequality is a consequence of our assumption above.

The perturbation argument we have used in 1.C still apply in the present setting ; there exist a unique $S \subset\left\{Y_{j}\right\}$ such that we have the next relation

$$
\begin{aligned}
& \mu^{\star}\left(K_{X}+\tau\left(\Theta_{\alpha}-\Theta_{\min }\right)+\Theta_{L}-\Theta_{\min }\right)+(1+\tau) \widehat{\Lambda}_{\min } \equiv \\
& \equiv K_{\widehat{X}}+S+\sum_{j \in J}\left(\tau\left(a_{\alpha}^{j}-a_{\min }^{j}\right)+a_{L}^{j}-a_{\min }^{j}-a_{\widehat{X} / X}^{j}\right)\left[Y_{j}\right]+\tau \widehat{\Lambda}_{\alpha}+\widehat{\Lambda}_{L}
\end{aligned}
$$

where the coefficients of $Y_{j}$ are strictly smaller than 1 , and the form $\widehat{\Lambda}_{L}$ is positively defined. The relation (83) is equivalent with

$$
\begin{aligned}
(1+\tau) \widehat{\Lambda}_{\min } & +\sum_{j \in J_{n}}\left(a_{\min }^{j}+a_{\widehat{X} / X}^{j}-\tau\left(a_{\alpha}^{j}-a_{\min }^{j}\right)-a_{L}^{j}\right)\left[Y_{j}\right] \equiv \\
& \equiv K_{\widehat{X}}+S+\sum_{j \in J_{p}}\left(\tau\left(a_{\alpha}^{j}-a_{\min }^{j}\right)+a_{L}^{j}-a_{\min }^{j}-a_{\widehat{X} / X}^{j}\right)\left[Y_{j}\right]+\tau \widehat{\Lambda}_{\alpha}+\widehat{\Lambda}_{L}
\end{aligned}
$$

We use the notation

$$
\widehat{L}:=\sum_{j \in J_{p}}\left(\tau\left(a_{\alpha}^{j}-a_{\min }^{j}\right)+a_{L}^{j}-a_{\min }^{j}-a_{\widehat{X} / X}^{j}\right)\left[Y_{j}\right]+\tau \widehat{\Lambda}_{\alpha}+\widehat{\Lambda}_{L}
$$


and we remark that $\widehat{L}$ is a big $\mathbb{R}$-line bundle on $\widehat{X}$, whose critical exponent is greater than 1 ; moreover, the restriction $\widehat{L}_{\mid S}$ has the same properties.

Next we invoke the non-vanishing theorem 0.1 : as a by-product of its proof, we get the family of approximations $\widehat{L}_{\eta}$ together with a corresponding family of effective Q-sections $\widehat{U}_{\eta}$ of the bundle

$$
K_{\widehat{X}}+S+\widehat{L}_{\eta}
$$

whose restriction to $S$ is non-zero, as they were obtained as extensions of non-zero sections defined on $S$. The bundle $K_{\widehat{X}}+S+\widehat{L}$ is obtained as a convex combination of the bundles of type (85), therefore we can assume the existence of a $\mathbb{R}$-divisor

$$
T:=\sum_{i \in I} \lambda^{i}\left[Z_{i}\right] \in\left\{K_{\widehat{X}}+S+\widehat{L}\right\}
$$

such that $\operatorname{card}(I)<\infty$ and such that $T$ is non-singular along $S$.

Then the current

$$
\begin{aligned}
\widehat{T} & :=T+\left((1+\tau) a_{\min }^{0}+a_{\widehat{X} / X}^{0}\right)[S]+ \\
& +\sum_{j \in J_{p}}\left((1+\tau) a_{\min }^{j}+a_{\widehat{X} / X}^{j}\right)\left[Y_{j}\right]+\sum_{j \in J_{n}}\left(\tau a_{\alpha}^{j}+a_{L}^{j}\right)\left[Y_{j}\right]
\end{aligned}
$$

belong to the class of the bundle

$$
K_{\widehat{X} / X}+(1+\tau) \mu^{\star}\left(K_{X}+L\right)
$$

where the index $j=0$ in (83) corresponds to $S$. By the Hartogs principle, we obtain

$$
\widehat{T}=(1+\tau) \mu^{\star} \Theta+\sum_{j \in J} a_{\widehat{X} / X}^{j}\left[Y_{j}\right]
$$

where $\Theta$ is an effective $\mathbb{R}$-divisor in the Chern class of $K_{X}+L$.

The current $\Theta$ is the one we seek ; it is obvious that the Lelong number of $\mu^{\star}(\Theta)$ along $S$ above is precisely the same as the Lelong number of the inverse image of the current $\Theta_{\min }$. The rationality statement in 2.B.1 can be obtained as in [39] : we write

$$
\mu^{\star}(\Theta)=a_{\min }^{0}[S]+\sum_{j} a_{\Theta}^{j}\left[W_{j}\right] \in \mu^{\star}\left(c_{1}\left(K_{X}+L\right)\right)
$$

where $W_{j} \subset \widehat{X}$ are hypersurfaces and $a_{\Theta}^{j}$ are positive real numbers.

If $a_{\text {min }}^{0} \notin \mathbb{Q}$, then we use the rationality of $L$ and infer the existence of an effective $\mathbb{Q}$-divisor

$$
b^{0} S+\sum_{j} b^{j} W_{j} \in \mu^{\star}\left(c_{1}\left(K_{X}+L\right)\right)
$$

such that $b^{0}<a_{\text {min }}^{0}$ (see [39] for a more complete discussion). The multiplication of the above $\mathbb{Q}$-section with a divisible enough positive integer transform it into a section of

$$
m_{0}\left(K_{X}+L\right)+\rho_{0}
$$

where $\rho_{0} \rightarrow X$ is a topologically trivial line bundle. By the theorem 2.A.1, the minimal metric on $K_{X}+L$ constructed with the sections of multiples of $K_{X}+L$ coincide with the minimal metric associated to the family of sections of the multiples of $m_{0}\left(K_{X}+L\right)+\rho_{0}$, thus we get a contradiction, and the theorem 2.B.1 is completely proved. 


\section{References}

[1] Bedford, E., Taylor.: The Dirichlet problem for a complex Monge-Ampère equation ; Invent. Math. 37 (1976), no. 1, 1-44.

[2] Berndtsson, B.: On the Ohsawa-Takegoshi extension theorem ; Ann. Inst. Fourier (1996).

[3] Berndtsson, B., Păun, M.: Bergman kernels and the pseudo-effectivity of the relative canonical bundles ; arXiv:math/0703344, to appear in Duke Math. Journal.

[4] Berndtsson, B., Păun, M.: A Bergman kernel proof of the Kawamata subadjunction theorem, I and $I I$; in preparation.

[5] Birkar, C., Cascini, P., Hacon, C., McKernan, J.: Existence of minimal models for varieties of log general type; on the web.

[6] Bonavero, L.: Inégalités de morse holomorphes singulières. (French) [Singular holomorphic Morse inequalities] ; J. Geom. Anal. 8 (1998), no. 3, 409-425.

[7] Boucksom, S. Divisorial Zariski decompositions on compact complex manifolds ; Ann. Sci. Ecole Norm. Sup. (4) 37 (2004), no. 1, 45-76.

[8] Campana, F., Peternell, Th. Geometric stability of the cotangent bundle and the universal cover of a projective manifold ; arXiv: math/0405093.

[9] Claudon, B.: Invariance for multiples of the twisted canonical bundle; math.AG/0511736, Ann. Inst. Fourier (Grenoble) 57 (2007), no. 1, 289-300.

[10] Corti, A.: Flips for 3-folds and 4-folds ; Oxford Lecture Ser. Math. Appl. 35(2007), 189 pp, Oxford Univ. Press.

[11] Demailly, J.-P.: Regularization of closed positive currents and Intersection Theory ; J. Alg. Geom. 1 (1992) 361-409.

[12] Demailly, J.-P.: A numerical criterion for very ample line bundles ; J. Differential Geom. 37 (1993), no. 2, 323-374.

[13] Demailly, J.-P.: On the Ohsawa-Takegoshi-Manivel extension theorem ; Proceedings of the Conference in honour of the 85th birthday of Pierre Lelong, Paris, September 1997, Progress in Mathematics, Birkauser, 1999.

[14] Demailly, J.-P., Peternell, Th., Schneider, M. : Pseudoeffective line bundles on compact Kähler manifolds ; Internat. J. Math. 12 (2001), no. 6.

[15] Demailly, J.-P.: Kähler manifolds and transcendental techniques in algebraic geometry ; Plenary talk and Proceedings of the Internat. Congress of Math., Madrid (2006), 34p, volume I.

[16] Druel, S.: Existence de modèles minimaux pour les variétés de type général ; Exposé 982 , Séminaire Bourbaki, 2007/08.

[17] Ein, L., Lazarsfeld, R., Mustaţă, M., Nakamaye, M., Popa, M. :Asymptotic invariants of base loci ; Ann. Inst. Fourier (Grenoble) 56 (2006), no. 6, 1701-1734.

[18] Ein, L., Popa, M.: Adjoint ideals and extension theorems ; preprint in preparation ; june 2007.

[19] Hacon, C., McKernan, J.: Boundedness of pluricanonical maps of varieties of general type ; Invent. Math. Volume 166, Number 1 / October, 2006, 1-25.

[20] Hacon, C., McKernan, J.: On the existence of flips ; math.AG/0507597.

[21] Hardy, G.H., Wright, E.M.: An introduction to the theory of numbers ; Oxford University Press, 1938.

[22] Kawamata, Y.: A generalization of Kodaira-Ramanujams vanishing theorem ; Math. Ann. 261 (1982), 4346.

[23] Kawamata, Y.: Pluricanonical systems on minimal algebraic varieties ; Invent. Math. 79 (1985), no. 3.

[24] Kawamata, Y.: On the extension problem of pluricanonical forms ; Contemporary. Math. 241 (1999), no. 3.

[25] Kawamata, Y.: Finite generation of a canonical ring ; arXiv:0804.3151.

[26] Kim, Dano.:Ph.D. Thesis ; Princeton, 2006.

[33] Kollár, J., Mori, S.:Birational geometry of algebraic varieties. ; Cambridge University Press, Cambridge, 1998. 
[28] Lazarsfeld, R.: Positivity in Algebraic Geometry ; Springer, Ergebnisse der Mathematik und ihrer Grenzgebiete.

[29] McNeal, J., Varolin, D.:Analytic inversion of adjunction: $L^{2}$ extension theorems with gain ; Ann. Inst. Fourier (Grenoble) 57 (2007), no. 3, 703-718.

[30] Nadel, A. M.: Multiplier ideal sheaves and Kahler-Einstein metrics of positive scalar curvature ; Ann. of Math. (2) 132 (1990), no. 3, 549596.

[31] Nakayama, N.:Zariski decomposition and abundance; MSJ Memoirs 14, Tokyo (2004).

[32] Ohsawa, T., Takegoshi, K. : On the extension of $L^{2}$ holomorphic functions ; Math. Z., 195 (1987), 197-204.

[33] Păun, M.: Siu's Invariance of Plurigenera: a One-Tower Proof ; preprint IECN (2005), J. Differential Geom. 76 (2007), no. 3, 485493.

[34] Shokurov, V.: A non-vanishing theorem ; Izv. Akad. Nauk SSSR (49) 1985.

[35] Skoda, H.: Sous-ensembles analytiques d'ordre fini ou infini dans $C^{n}$ (French) ; Bull. Soc. Math. France 100 (1972), 353-408.

[36] Siu, Y.-T.: Analyticity of sets associated to Lelong numbers and the extension of closed positive currents Invent. Math. 27 (1974), 53-156.

[37] Siu, Y.-T.: Invariance of Plurigenera ; Inv. Math., 134 (1998), 661-673.

[38] Siu, Y.-T.: Extension of twisted pluricanonical sections with plurisubharmonic weight and invariance of semipositively twisted plurigenera for manifolds not necessarily of general type ; Complex geometry (Göttingen, 2000), 223-277, Springer, Berlin, 2002.

[39] Siu, Y.-T.: A General Non-Vanishing Theorem and an Analytic Proof of the Finite Generation of the Canonical Ring; arXiv:math/0610740.

[40] Siu, Y.-T.: Finite Generation of Canonical Ring by Analytic Method ; arXiv:0803.2454.

[41] Takayama, S: On the Invariance and Lower Semi-Continuity of Plurigenera of Algebraic Varieties ; J. Algebraic Geom. 16 (2007), no. 1, 1-18.

[42] Takayama, S: Pluricanonical systems on algebraic varieties of general type ;Invent. Math. Volume 165, Number 3 / September, 2005, 551-587.

[43] Tsuji, H.: Extension of log pluricanonical forms from subvarieties; math.CV/0511342.

[44] Varolin, D.: A Takayama-type extension theorem; math.CV/0607323, to appear in Comp. Math.

[45] Viehweg, E.: Vanishing theorems ; J. Reine Angew. Math. 335 (1982).

[46] Yau, S-T.: On the Ricci curvature of a compact $K$ ähler manifold and the complex MongeAmpère equation. I.; Comm. Pure Appl. Math. 31 (1978), no. 3, 339411.

(version of July 19, 2008, printed on November 11, 2018)

Mihai Păun, paun@iecn.u-nancy.fr 